\title{
Meromorphic continuation of Koba-Nielsen string amplitudes
}

\author{
M. Bocardo-Gaspar, ${ }^{a}$ Willem Veys ${ }^{b}$ and W.A. Zúñiga-Galindo ${ }^{c, 1}$ \\ ${ }^{a}$ Departamento de Matemáticas, CUCEI, Universidad de Guadalajara, \\ Blvd. Marcelino García Barragán \#1421, Guadalajara, Jal. 44430, México \\ ${ }^{b}$ Department of Mathematics, Katholieke Universiteit Leuven, \\ Celestijnenlaan 200 B, B-3001 Leuven, Belgium \\ ${ }^{c}$ Departamento de Matemáticas, Unidad Querétaro, \\ Centro de Investigación y de Estudios Avanzados del Instituto Politécnico Nacional, \\ Libramiento Norponiente \#2000, Fracc. Real de Juriquilla. Santiago de Querétaro, \\ Qro. 76230, México \\ E-mail: miriam.bocardo@academicos.udg.mx, wim.veys@kuleuven.be, \\ wazuniga@math. cinvestav.edu.mx
}

ABSTRACT: In this article, we establish in a rigorous mathematical way that Koba-Nielsen amplitudes defined on any local field of characteristic zero are bona fide integrals that admit meromorphic continuations in the kinematic parameters. Our approach allows us to study in a uniform way open and closed Koba-Nielsen amplitudes over arbitrary local fields of characteristic zero. In the regularization process we use techniques of local zeta functions and embedded resolution of singularities. As an application we present the regularization of $p$-adic open string amplitudes with Chan-Paton factors and constant $B$-field. Finally, all the local zeta functions studied here are partition functions of certain $1 D \log$-Coulomb gases, which shows an interesting connection between Koba-Nielsen amplitudes and statistical mechanics.

KEywords: Bosonic Strings, D-branes, Differential and Algebraic Geometry

ARXIV EPRINT: 1905.10879

\footnotetext{
${ }^{1}$ The second author was supported by KU Leuven grant C14/17/083. The third author was partially supported by Conacyt Grant No. 250845.
} 


\section{Contents}

1 Introduction 2

2 Discussion of the results $\quad 6$

2.1 Open string tree amplitudes with Chan-Paton factors $\quad 7$

2.2 Koba-Nielsen local zeta functions and $1 D$ log-Coulomb gases 10

3 Multivariate local zeta functions and embedded resolution of singularities

4 Local zeta functions of Koba-Nielsen type

5 Road map of the proof $\quad 16$

$\begin{array}{lll}5.1 \text { Example } & 17\end{array}$

$\begin{array}{lll}5.2 & \text { Example } & 17\end{array}$

5.3 Case $I=\{2, \ldots, N-2\} \quad 19$

5.4 Case $I \neq\{2, \ldots, N-2\}$

5.5 Proof of Theorem 4.1 and precise description of the convergence domain 26

$\begin{array}{ll}5.6 \text { Example } & 27\end{array}$

6 Local zeta functions of Koba-Nielsen type over local fields 28

$\begin{array}{lll}6.1 \text { Local fields } & 28\end{array}$

6.2 Multivariate local zeta functions: general case 28

6.3 Meromorphic continuation of local zeta functions: general case 30

6.4 A result of Vanhove and Zerbini 31

7 Meromorphic continuation of Koba-Nielsen string amplitudes over local fields of characteristic zero

7.1 Convergence of the Koba-Nielsen amplitudes 33

7.2 Meromorphic continuation of Koba-Nielsen string amplitudes 34

$\begin{array}{lll}7.3 & \text { Tachyon scattering } & 35\end{array}$

$\begin{array}{lll}\text { 7.3.1 Example } & 37\end{array}$

8 Amplitudes and gamma functions $\quad 37$

$\begin{array}{ll}8.1 \text { Veneziano amplitude } & 37\end{array}$

$\begin{array}{lll}8.2 & A_{\mathbb{R}}^{(N)}(\boldsymbol{k}) \text { as a sum of gamma functions } & 38\end{array}$ 


\section{Introduction}

In the recent years, scattering amplitudes, considered as mathematical structures, have been studied intensively, see e.g. $[3,26]$ and the references therein. The main motivations driving this research are, from one side, the development of more efficient methods to calculate amplitudes, and on the other side, the existence of deep connections with many mathematical areas, among them, algebraic geometry, combinatorics, number theory, $p$ adic analysis, etc., see e.g. [7, 11, 12, 14-22, 27-30, 32-34, 45, 47-51], and the references therein. In the 80 s the idea that string amplitudes at the tree level can be studied over different number fields and that there are connections between these amplitudes emerged in the works of Freund, Witten and Volovich, among others, see e.g. [18, 19, 54]. In this framework the connection with local zeta functions appears naturally. The present work is framed in the 'emerging idea' that scattering amplitudes are local zeta functions in the sense of Gel'fand, Weil, Igusa, Sato, Bernstein, Denef, Loeser, etc., and also it continues our investigation of the connections between string amplitudes at the tree level and local zeta functions $[11,12,30]$.

In this article we establish, in a rigorous mathematical way, that the Koba-Nielsen string amplitudes defined on any local field of characteristic zero are bona fide integrals. Furthermore, they admit extensions which are meromorphic complex functions in the kinematic parameters. We express the Koba-Nielsen amplitudes as linear combinations of multivariate local zeta functions, and, by using embedded resolution of singularities (Hironaka's theorem [35]), we show that all these local zeta functions are holomorphic in a common domain, and then we use the fact that the local zeta functions admit meromorphic continuations. Since Hironaka's theorem is valid over any field of characteristic zero, we are able to regularize the Koba-Nielsen amplitudes defined over $\mathbb{R}, \mathbb{C}$, or $\mathbb{Q}_{p}$, the field of $p$-adic numbers, at the same time.

We denote by $\mathbb{K}$ a local field of characteristic zero, and set $\boldsymbol{f}:=\left(f_{1}, \ldots, f_{m}\right)$ and $s:=\left(s_{1}, \ldots, s_{m}\right) \in \mathbb{C}^{m}$, where the $f_{i}(x)$ are non-constant polynomials in the variables $x:=\left(x_{1}, \ldots, x_{n}\right)$ with coefficients in $\mathbb{K}$. The multivariate local zeta function attached to $(\boldsymbol{f}, \Theta)$, where $\Theta$ is a test function, is defined as

$$
Z_{\Theta}(\boldsymbol{f}, \boldsymbol{s})=\int_{\mathbb{K}^{n}} \Theta(x) \prod_{i=1}^{m}\left|f_{i}(x)\right|_{\mathbb{K}}^{s_{i}} \prod_{i=1}^{n} d x_{i}, \quad \text { when } \operatorname{Re}\left(s_{i}\right)>0 \text { for all } i
$$

and where $\prod_{i=1}^{n} d x_{i}$ is the normalized Haar measure of $\left(\mathbb{K}^{n},+\right)$. These integrals admit meromorphic continuations to the whole $\mathbb{C}^{m},[37,38,46]$, see also $[31,39]$. In the 60 s, Weil studied local zeta functions, in the Archimedean and non-Archimedean settings, in connection with the Poisson-Siegel formula. In the 70s, Igusa developed a uniform theory for local zeta functions in characteristic zero [37, 38], see also [46, 58, 59]. In the $p$-adic setting, the local zeta functions are connected with the number of solutions of polynomial congruences $\bmod p^{l}$ and with exponential sums $\bmod p^{l}[23]$. More recently, Denef and Loeser introduced the motivic zeta functions which constitute a vast generalization of $p$ adic local zeta functions [24]. 
In the case $\mathbb{K}=\mathbb{R}$ and $m=1$, the local zeta functions were introduced in the 50s by Gel'fand and Shilov. The main motivation was that the meromorphic continuation of Archimedean local zeta functions implies the existence of fundamental solutions (i.e. Green functions) for differential operators with constant coefficients. This fact was established, independently, by Atiyah [5] and Bernstein [9]. It is important to mention here that, in the $p$-adic framework, the existence of fundamental solutions for pseudodifferential operators is also a consequence of the fact that the Igusa local zeta functions admit a meromorphic continuation, see ([60], chapter 5) ([44], chapter 10). This analogy turns out to be very important in the rigorous construction of quantum scalar fields in the $p$-adic setting, see [48] and the references therein.

The connections between Feynman amplitudes and local zeta functions are very old and deep. Let us mention that the works of Speer [50] and Bollini, Giambiagi and González Domínguez [17] on regularization of Feynman amplitudes in quantum field theory are based on the analytic continuation of distributions attached to complex powers of polynomial functions in the sense of Gel'fand and Shilov [31], see also [7, 8, 16, 47], among others. The book [31], which is one of the main sources for the ' $i \epsilon$ regularization method' widely used, was written before the establishing of Hironaka's theorem [35]. After the work of Atiyah, Bernstein and Igusa, among others, the $i \epsilon$ regularization method was substituted by the embedded resolution of singularities technique, see [37, 38]. However, this method is not widely used by theoretical physicists. In [57] Witten discusses the classical $i \epsilon$ regularization method for string amplitudes; in this article, we present a rigorous regularization of the Koba-Nielsen string amplitudes using the 'modern $i \epsilon$ regularization method'.

Take $N \geq 4$, and complex variables $s_{1 j}$ and $s_{(N-1) j}$ for $2 \leq j \leq N-2$ and $s_{i j}$ for $2 \leq i<j \leq N-2$. Put $s:=\left(s_{i j}\right) \in \mathbb{C}^{\boldsymbol{d}}$, where $\boldsymbol{d}=\frac{N(N-3)}{2}$ denotes the total number of indices $i j$. In this article we introduce the multivariate local zeta function

$$
Z_{\mathbb{K}}^{(N)}(s):=\int_{\mathbb{K}^{N-3}} \prod_{i=2}^{N-2}\left|x_{j}\right|_{\mathbb{K}}^{s_{1 j}}\left|1-x_{j}\right|_{\mathbb{K}}^{s_{(N-1) j}} \prod_{2 \leq i<j \leq N-2}\left|x_{i}-x_{j}\right|_{\mathbb{K}}^{s_{i j}} \prod_{i=2}^{N-2} d x_{i}
$$

where $\prod_{i=2}^{N-2} d x_{i}$ is the normalized Haar measure on $\mathbb{K}^{N-3}$. We have called integrals of type (1.1) Koba-Nielsen local zeta functions. These functions have a statistical mechanics interpretation as partition functions of certain $1 D$ log-Coulomb gases, see section 2.2.

We show that these functions are bona fide integrals, which are convergent and holomorphic in an open part of $\mathbb{C}^{\mathbf{d}}$, containing the set given by $\frac{-2}{N-2}<\operatorname{Re}\left(s_{i j}\right)<\frac{-2}{N}$ for all $i j$. Furthermore, they admit meromorphic continuations to the whole $\mathbb{C}^{d}$, see Theorems 6.1 and 4.1. We give a detailed proof and a precise description of the convergence domain in the case $\mathbb{K}=\mathbb{R}$, see Theorem 4.1 and section 5; this proof can be easily extended to an arbitrary local field $\mathbb{K}$ of characteristic zero, see section 6 .

The Koba-Nielsen open string amplitudes for $N$-points over $\mathbb{K}$ are formally defined as

$$
A_{\mathbb{K}}^{(N)}(\boldsymbol{k}):=\int_{\mathbb{K}^{N-3}} \prod_{i=2}^{N-2}\left|x_{j}\right|_{\mathbb{K}}^{\boldsymbol{k}_{1} \boldsymbol{k}_{j}}\left|1-x_{j}\right|_{\mathbb{K}}^{\boldsymbol{k}_{N-1} \boldsymbol{k}_{j}} \prod_{2 \leq i<j \leq N-2}\left|x_{i}-x_{j}\right|_{\mathbb{K}}^{\boldsymbol{k}_{i} \boldsymbol{k}_{j}} \prod_{i=2}^{N-2} d x_{i}
$$


where $\boldsymbol{k}=\left(\boldsymbol{k}_{1}, \ldots, \boldsymbol{k}_{N}\right), \boldsymbol{k}_{i}=\left(k_{0, i}, \ldots, k_{l, i}\right) \in \mathbb{R}^{l+1}$, for $i=1, \ldots, N(N \geq 4)$, is the momentum vector of the $i$-th tachyon (with Minkowski product $\boldsymbol{k}_{i} \boldsymbol{k}_{j}=-k_{0, i} k_{0, j}+k_{1, i} k_{1, j}+$ $\left.\cdots+k_{l, i} k_{l, j}\right)$, obeying

$$
\sum_{i=1}^{N} \boldsymbol{k}_{i}=\mathbf{0}, \quad \boldsymbol{k}_{i} \boldsymbol{k}_{i}=2 \text { for } i=1, \ldots, N .
$$

The parameter $l$ is an arbitrary positive integer. Typically $l$ is taken to be 25 . However, we do not require using the critical dimension. We choose units such that the tachyon mass is $m^{2}=-2$. To carry out a mathematical study of the integral (1.2) it is more convenient to take $\boldsymbol{k}_{i} \boldsymbol{k}_{j}=s_{i j} \in \mathbb{C}$. Several different problems occur depending whether or not the $\boldsymbol{k}_{i}$ are real or complex and if the kinematic restrictions (1.3) are considered or not.

The amplitudes of type (1.2) were introduced in the works of Brekke, Freund, Olson and Witten, among others, on string theory in the adelic framework, see e.g. ([19], section 8). We use here all the conventions introduced in [19]. In the real case, $A_{\mathbb{R}}^{(N)}(\boldsymbol{k})$ is (up to multiplication by a positive constant) the open Koba-Nielsen amplitude of $N$-points, see ([19], section 8), ([40], section 2). If $N=4, A_{\mathbb{R}}^{(4)}(\boldsymbol{k})$ is the Veneziano amplitude [52]. In the complex case, $A_{\mathbb{C}}^{(N)}(\boldsymbol{k})$ is just a mathematical object. However, by using the results of ([40], section 2), see also [10], the $N$-point, closed string amplitude at the tree level is the product of $A_{\mathbb{C}}^{(N)}(\boldsymbol{k})$ times a polynomial function in the momenta $\boldsymbol{k}$. This fact implies that our techniques and results are applicable to classical closed string amplitudes at the tree level.

A central problem is to know whether or not integrals of type (1.2) converge for some values of $\boldsymbol{k}$. We use the integrals $Z_{\mathbb{K}}^{(N)}(s)$ as regularizations of the amplitudes $A_{\mathbb{K}}^{(N)}(\boldsymbol{k})$. More precisely, we redefine

$$
A_{\mathbb{K}}^{(N)}(\boldsymbol{k})=\left.Z_{\mathbb{K}}^{(N)}(\boldsymbol{s})\right|_{s_{i j}=\boldsymbol{k}_{i} \boldsymbol{k}_{j}},
$$

where $Z_{\mathbb{K}}^{(N)}(s)$ now denotes the meromorphic continuation of (1.1) to the whole $\mathbb{C}^{\boldsymbol{d}}$, see Theorem 6.1.

We show that $A_{\mathbb{K}}^{(N)}(\boldsymbol{k})$ converges on some open in $\mathbb{C}^{(N-1)(l+1)}$ by showing that this open is mapped into the domain of convergency of $Z_{\mathbb{K}}^{(N)}(\boldsymbol{s})$ by $\boldsymbol{k} \rightarrow s_{i j}=\boldsymbol{k}_{i} \boldsymbol{k}_{j}$. Our Theorem 7.1 establishes further that $A_{\mathbb{K}}^{(N)}(\boldsymbol{k})$ extends to a meromorphic function to the whole $\mathbb{C}^{(N-1)(l+1)}$, and that its polar set is contained in the inverse image of the polar set of $Z_{\mathbb{K}}^{(N)}(s)$ under that mapping. Also, it describes the possible poles using numerical data of suitable resolutions of singularities. It is important to mention here that, in the regularization of $A_{\mathbb{K}}^{(N)}(\boldsymbol{k})$, we do not use the kinematic restrictions (1.3). On the other hand, in the cases $\mathbb{K}=\mathbb{R}, \mathbb{C}$, the meromorphic continuation of $A_{\mathbb{K}}^{(N)}(\boldsymbol{k})$ can be given in terms of gamma functions, see section 8.2.

As an illustration, we describe in the cases $N=4,5,6$ explicitly the convergence domain of $Z_{\mathbb{K}}^{(N)}(s)$, see Examples 5.1, 5.2, 5.6. In the case $N=4, A_{\mathbb{R}}^{(4)}(\boldsymbol{k})$ is exactly the Veneziano amplitude [52]. It is well-known that $A_{\mathbb{R}}^{(4)}(\boldsymbol{k})$ can be expressed as a sum of gamma functions, and the domain of convergence of the integral $A_{\mathbb{R}}^{(4)}(\boldsymbol{k})$ is known. Our Theorem 7.1 gives exactly this domain of convergence, see section 8.1. 
Our methods allow in principle to express $A_{\mathbb{R}}^{(N)}(\boldsymbol{k})$ as a sum of monomial integrals and as a linear combination of gamma functions, with coefficients in the algebra of holomorphic functions on $\mathbb{C}^{(N-1)(l+1)}$, see section 8.2. By using the result of Example 5.2 (and 5.6), this could be carried out explicitly for $N=5$ (and $N=6$ ).

The study of the scattering process of $N$-tachyons with momenta $\boldsymbol{k}_{1}, \ldots, \boldsymbol{k}_{N} \in \mathbb{R}^{l+1}$, each of them with mass $m^{2}=-2$, requires determining a value of the meromorphic function $A_{\mathbb{K}}^{(N)}(\boldsymbol{k})$ for $\boldsymbol{k}$ belonging to the real algebraic set (1.3). In this framework, $\boldsymbol{k}_{i} \boldsymbol{k}_{i}=2$ is the relativistic energy for the $i$-th tachyon. Then, increasing $N$ means to increase the energy of the scattering process. Our efforts for finding real solutions of (1.3) contained in the domain of convergence of the integral $A_{\mathbb{K}}^{(N)}(\boldsymbol{k})$ suggest that it is unlikely to find such solutions for large $N$. In contrast, if $N \leq l+1$, we show that the restriction of $A_{\mathbb{K}}^{(N)}(\boldsymbol{k})$ to the set (1.3) gives rise to a well-defined scattering amplitude, see Proposition 7.2.

On the other hand, for $N>l+1$, the existence of points of the algebraic set (1.3) that are not contained in the polar locus of $A_{\mathbb{K}}^{(N)}(\boldsymbol{k})$ is a non-trivial question. For instance, in the basic case $N=4$ and $l=1$, such points do not exist. As soon as $l \geq 2$, we do expect that complex solutions exist, and we constructed some for infinitely many $N$, see Example 7.3.1.

In the case $A_{\mathbb{K}}^{(N)}(\boldsymbol{k})=\infty$, the determination of a scattering amplitude requires a renormalization process for the meromorphic amplitude $A_{\mathbb{K}}^{(N)}(\boldsymbol{k})$. One can extend the function on the part of (1.3) outside the polar locus, or proceed via the computation of Laurent series, which are available in this case, see section 8.2.

Our results on Koba-Nielsen local zeta functions can be easily extended to the case in which the integrals defining them contain multiplicative characters. In section 2.1, we show that the techniques introduced here allow us to regularize general $p$-adic open string amplitudes with Chan-Paton rules and a constant $B$-field. The regularization problem of these integrals was posed in [30], see also [32].

The string amplitudes were introduced by Veneziano in the 60s, [52], further generalizations were obtained by Virasoro [53], Koba and Nielsen [43], among others. The $p$-adic string amplitudes emerged in the 80s in the works of Freund and Olson [28], Freund and Witten [29], see also [18], Frampton and Okada [27], and Volovich [54]. Since the 60s the string amplitudes at the tree level have been used as formal objects in many physical calculations. In [11], it was established in the $p$-adic setting and by using techniques of Igusa's local zeta functions that the Koba-Nielsen amplitudes are bona fide integrals. In this article this result is extended to an arbitrary local field of characteristic zero. We show that the open and closed string amplitudes at the tree level can be studied in a uniform way on any local field of characteristic zero, see Theorems 6.1, 7.1. This is consistent with Volovich's conjecture asserting that the mathematical description of physical reality must not depend on the background number field, see [55]. 


\section{Discussion of the results}

After the publication of this manuscript in arxiv.org, we became aware of two other manuscripts, also available in arxiv.org, containing some similar results, see [22, 51]. Indeed, we are dealing here with some matters considered in the mentioned works, but our perspective, problems, and techniques are completely different, and mainly the main results of all these works are complementary. Here we study string amplitudes at the tree level as algebraic-arithmetic objects over arbitrary local fields of characteristic zero. This implies that we can not use presentations of the amplitudes that involve the standard real order. In addition, we do not use the Mandelstam variables. In contrast, in [22, 51], the classical string amplitudes are studied using moduli algebraic-geometric techniques. Then the results about the convergence of the amplitudes (see ([22], Propositions 3.5 and 3.6), ([51], Proposition 7.2)) presented in these works cannot be compared directly with our results, see e.g. Propositions 7.1, 7.2.

Our Theorem 7.1 establishes the existence of a meromorphic continuation for $A_{\mathbb{K}}^{(N)}(\boldsymbol{k})$ in the kinematic parameters. The meromorphic continuation provides more than a regularization of the original amplitude; it shows in particular that the original integral converges on some open region. The polar set of $A_{\mathbb{K}}^{(N)}(\boldsymbol{k})$ contains all the information on the ultraviolet and infrared divergencies of the amplitude. Furthermore, in the case $\mathbb{K}=\mathbb{R}, \mathbb{C}$, we show that $A_{\mathbb{K}}^{(N)}(\boldsymbol{k})$ is a linear combination of gamma functions with coefficients in the ring of holomorphic functions on $\mathbb{C}^{(N-1)(l+1)}$. The above-mentioned works do not contain similar results. Theorem 7.1 also implies the existence of Laurent expansions for the string amplitudes in the kinematic parameters.

On the other hand, the main result of [22] is a regularization of both open and closed string perturbation amplitudes at tree level. Furthermore, the amplitudes admit Laurent expansion in Mandelstam variables whose coefficients are multiple zeta values (resp. singlevalued multiple zeta values). Then, in ([22], Theorems 4.20 and 4.24) a very general theory of Laurent series for string amplitudes is presented, which gives a detailed local description of the string amplitudes. In contrast, here we provide a global description of the open/closed string amplitudes as meromorphic functions, but we do not provide a detailed description of the coefficients of the Laurent expansions.

In section 6.4 we discuss a result of Vanhove and Zerbini ([51], Proposition 7.2) on the convergence of the integrals $Z_{\mathbb{C}}^{(N)}(s)$. The authors use an ad hoc procedure (partially also using changes of variables of 'blow-up type') to study the convergence of these integrals. However, we claim that their stated convergence domain is too large, in the sense that some necessary inequalities were forgotten. We illustrate this in particular for the case $N=5$, giving a concrete $s_{0}$ in the stated convergence domain of ([51], Proposition 7.2) for which $Z_{\mathbb{C}}^{(5)}\left(s_{0}\right)$ diverges. In our view, this confirms that the technique of resolution of singularities is a very appropriate tool to study convergence of Koba-Nielsen string amplitudes.

We also consider the problem of determining the scattering amplitude for the interaction of $N$ tachyons, cf. Proposition 7.2, which is not discussed in [22, 51]. Finally, our techniques can be used to study $p$-adic open string amplitudes, with Chan-Paton rules and a constant $B$-field, and the local zeta functions introduced here have a statistical mechanics interpretation. In the next two sections we discuss these last matters. 


\subsection{Open string tree amplitudes with Chan-Paton factors}

The connections between noncommutative geometry and string theory are very deep and relevant, at mathematical and physical levels. The study of the 'Koba-Nielsen string amplitudes' coming from these theories is a relevant matter. The mathematical framework developed here allows us naturally to study these amplitudes. The study of such amplitudes is out of scope of $[22,51]$.

In ordinary string theory, the effective action for bosonic open strings in gauge field backgrounds was discussed many years ago in [1]. The analysis incorporating a NeveuSchwarz $B$-field in the target space leads to a noncommutative effective gauge theory on the world-volume of D-branes [49]. The study of the $p$-adic open string tree amplitudes including Chan-Paton factors was started in [18]. However, the incorporation of a $B$-field in the $p$-adic context and the computation of the tree level string amplitudes was discussed in $[32,34]$. In these works it was reported that the tree-level string amplitudes are affected by a noncommutative factor. In [32] Ghoshal and Kawano introduced new amplitudes involving multiplicative characters and a noncommutative factor. These amplitudes coincide with the ones obtained directly from the noncommutative effective action [33].

In [30] the regularization of the $p$-adic open string amplitudes, with Chan-Paton rules and a constant $B$-field, introduced by Ghoshal and Kawano, was established rigorously. The authors use techniques of multivariate local zeta functions depending on multiplicative characters and a phase factor which involves an antisymmetric bilinear form. By attaching to each amplitude a multivariate local zeta function depending on the kinematic parameters, the $B$-field and the Chan-Paton factors, the authors show that these integrals admit meromorphic continuations in the kinematic parameters.

We define the $N$-point, open string amplitude, with Chan-Paton rules in a constant $B$-field over $\mathbb{K}$, by

$A_{\mathbb{K}}^{(N)}\left(\boldsymbol{k}, \theta, \operatorname{sgn}_{\mathbb{K}}\right)=$

$\int_{\mathbb{K}^{N}} \prod_{1 \leq i<j \leq N}\left|x_{i}-x_{j}\right|_{\mathbb{K}}^{\boldsymbol{k}_{i} \boldsymbol{k}_{j}} H_{\mathbb{K}}\left(x_{i}-x_{j}\right) \exp \left\{-\frac{\sqrt{-1}}{2}\left(\sum_{1 \leq i<j \leq N}\left(\boldsymbol{k}_{i} \theta \boldsymbol{k}_{j}\right) \operatorname{sgn}_{\mathbb{K}}\left(x_{i}-x_{j}\right)\right)\right\} \prod_{i=1}^{N} d x_{i}$,

where $N \geq 4, \boldsymbol{k}=\left(\boldsymbol{k}_{1}, \ldots, \boldsymbol{k}_{N}\right), \boldsymbol{k}_{i}=\left(k_{0, i}, \ldots, k_{l, i}\right), i=1, \ldots, N$, is the momentum vector of the $i$-th tachyon vertex operator obeying $(1.3), H_{\mathbb{K}}(x)=\frac{1}{2}\left(1+\operatorname{sgn}_{\mathbb{K}}(x)\right), \operatorname{sgn}_{\mathbb{K}}(x)$ is a $\mathbb{K}$-version of the sign function, $\theta$ is a fixed antisymmetric bilinear form, and $\prod_{i=1}^{N} d x_{i}$ is the normalized Haar measure on $\left(\mathbb{K}^{N},+\right)$. These amplitudes are non-commutative generalizations of the Koba-Nielsen amplitudes. Indeed, the non-commutativity comes from the fact that $\boldsymbol{k}_{j} \theta \boldsymbol{k}_{i} \neq \boldsymbol{k}_{i} \theta \boldsymbol{k}_{j}$, and by turning of the $B$-field, i.e., taking $\theta=0$ and $\operatorname{sgn}_{\mathbb{K}}=1$, one obtains an amplitude similar to (1.2) but in a different number of variables.

The functions $\operatorname{sgn}_{\mathbb{K}}$ are multiplicative characters of the multiplicative group $\left(\mathbb{K}^{\times}, \cdot\right)$. For any $\mathbb{K}$ there is a trivial character, $\operatorname{sgn}_{\mathbb{K}}(x)=1$ for any $x \in \mathbb{K}^{\times}$. In the case $\mathbb{K}=\mathbb{R}$, there is only one non-trivial multiplicative character, $\operatorname{sgn}_{\mathbb{R}}(x)=\frac{x}{|x|}, x \in \mathbb{R}^{\times}$; in the case $\mathbb{K}=\mathbb{C}$, the nontrivial multiplicative characters have the form $\operatorname{sgn}_{\mathbb{C}}(x)=\left(\frac{x}{|x|}\right)^{m}$, for some integer $m$, and $x \in \mathbb{C}^{\times}$. For the discussion of the $p$-adic case the reader may consult [30]. 
This type of theories is not invariant under projective Möbius transformations and consequently the normalization

$$
x_{1}=0, x_{N-1}=1, x_{N}=\infty
$$

can not be carried out. In [32], in the case $\mathbb{K}=\mathbb{Q}_{p}$, the authors assume such a normalization and study the corresponding amplitudes for some particular values of $N$. In [30], these amplitudes were studied for an arbitrary number of points, using resolution of singularities. One of the conclusions obtained there was that imposing the conformal gauge (2.2) produces amplitudes with strange properties, and consequently, the study of amplitudes of the form (2.1) is completely necessary. It is important to mention that integrals (2.1) appear in a $p$-adic framework, but since they are algebro-geometric objects, here we study them over arbitrary fields of characteristic zero.

As an application of the techniques and results presented here, we show implicitly also the existence of a meromorphic continuation for amplitudes of type (2.1), in the case $\mathbb{K}$ is $\mathbb{R}$ or a $p$-adic field. We attach to $A_{\mathbb{K}}^{(N)}\left(\boldsymbol{k}, \theta, \operatorname{sgn}_{\mathbb{K}}\right)$ the Igusa type integral

$$
Z_{\mathbb{K}}^{(N)}\left(\boldsymbol{s}, \widetilde{\boldsymbol{s}}, \operatorname{sgn}_{\mathbb{K}}\right):=\int_{\mathbb{K}^{N}} \prod_{1 \leq i<j \leq N}\left|x_{i}-x_{j}\right|_{\mathbb{K}}^{s_{i j}} H_{\mathbb{K}}\left(x_{i}-x_{j}\right) E\left(\boldsymbol{x}, \widetilde{\boldsymbol{s}}, \operatorname{sgn}_{\mathbb{K}}\right) \prod_{i=1}^{N} d x_{i},
$$

where

$$
E\left(\boldsymbol{x}, \widetilde{\boldsymbol{s}}, \operatorname{sgn}_{\mathbb{K}}\right):=\exp \left\{-\frac{\sqrt{-1}}{2}\left(\sum_{1 \leq i<j \leq N} \widetilde{s}_{i j} \operatorname{sgn}_{\mathbb{K}}\left(x_{i}-x_{j}\right)\right)\right\},
$$

the $s_{i j}$ are complex symmetric variables and the $\widetilde{s}_{i j}$ are real antisymmetric variables.

If $\mathbb{K}$ is $\mathbb{R}$ or a $p$-adic field, then $\operatorname{sgn}_{\mathbb{K}}(x) \in\{ \pm 1\}$ for $x \in \mathbb{K}^{\times}$, and then for $\widetilde{s} \in \mathbb{R}$ and $x \in \mathbb{K}^{\times}$,

$$
\exp \left(-\frac{\sqrt{-1} \widetilde{s}}{2} \operatorname{sgn}_{\mathbb{K}}(x)\right)=\cos \left(\frac{\widetilde{s}}{2}\right)-\sqrt{-1} \operatorname{sgn}_{\mathbb{K}}(x) \sin \left(\frac{\widetilde{s}}{2}\right) .
$$

By using this identity, we get

$$
E\left(\boldsymbol{x}, \widetilde{\boldsymbol{s}}, \operatorname{sgn}_{\mathbb{K}}\right)=\sum_{J} E_{J}(\widetilde{\boldsymbol{s}}) \prod_{i, j \in J ; i<j} \operatorname{sgn}_{\mathbb{K}}\left(x_{i}-x_{j}\right),
$$

where the $E_{J}(\widetilde{\boldsymbol{s}})$ are polynomial functions in the variables $\cos \left(\widetilde{s}_{i j}\right), \sin \left(\widetilde{s}_{i j}\right)$. In addition, we also have the identity

$$
\prod_{1 \leq i<j \leq N} H_{\mathbb{K}}\left(x_{i}-x_{j}\right)=c+\sum_{J} e_{J} \prod_{i, j \in J ; i<j} \operatorname{sgn}_{\mathbb{K}}\left(x_{i}-x_{j}\right),
$$

where $c$ and the $e_{J}$ are constants, and $J$ runs over a family of subsets of $\{1, \ldots, N\}$, see also ([30], section 5.1). By using these identities, $Z_{\mathbb{K}}^{(N)}\left(\boldsymbol{s}, \widetilde{\boldsymbol{s}}, \operatorname{sgn}_{\mathbb{K}}\right)$ is a linear combination of twisted Koba-Nielsen zeta functions of the form

$$
Z_{\mathbb{K}}^{(N)}(s, \chi ; J):=\int_{\mathbb{K}^{N}} \prod_{i, j \in J ; i<j}\left\{\left|x_{i}-x_{j}\right|_{\mathbb{K}}^{s_{i j}} \chi_{i j}\left(x_{i}-x_{j}\right)\right\} \prod_{i=1}^{N} d x_{i},
$$


where $\chi=\left(\chi_{i j}\right)_{i, j \in J}$ and $\chi_{i j}=\operatorname{sgn}_{\mathbb{K}}$ or $\chi_{i j}=1$. By using a partition of the unity of the form $1_{\mathbb{K}}=\sum_{I \subset\{1, \ldots, N\}} \Theta_{I}(\boldsymbol{x})$, where $\Theta_{I}$ is a smooth function (in the case $\mathbb{K}=\mathbb{R}$,) or a locally constant function (in the non-Archimedean case), see e.g. Definition $2, Z_{\mathbb{K}}^{(N)}(s, \chi ; J)$ becomes a linear combination of integrals of the form

$$
Z_{\mathbb{K}}^{(N)}\left(s, \chi ; \Theta_{J}, J\right):=\int_{\mathbb{K}^{N}} \Theta_{J}(\boldsymbol{x}) \prod_{i, j \in J ; i<j}\left\{\left|x_{i}-x_{j}\right|_{\mathbb{K}}^{s_{i j}} \chi_{i j}\left(x_{i}-x_{j}\right)\right\} \prod_{i=1}^{N} d x_{i} .
$$

These integrals are twisted multivariate local zeta functions if $\Theta_{J}$ has compact support.

By renaming the variables, we may assume without loss of generality that

$$
\prod_{i, j \in J ; i<j}\left\{\left|x_{i}-x_{j}\right|_{\mathbb{K}}^{s_{i j}} \chi_{i j}\left(x_{i}-x_{j}\right)\right\}=\prod_{l \leq i<j \leq n}\left\{\left|x_{i}-x_{j}\right|_{\mathbb{K}}^{s_{i j}} \chi_{i j}\left(x_{i}-x_{j}\right)\right\}
$$

for some integers $l, n$ satisfying $1 \leq l<n \leq N$. If $\Theta_{J}$ has an unbounded support, we may assume that there exists $m$ satisfying $1 \leq l \leq m \leq n \leq N$, such that $x_{i}$ runs through a bounded set for $i=l, \ldots, m-1$, and through a unbounded set for $i=m, \ldots, n$. By changing variables $x_{i} \rightarrow x_{i}$ for $i=l, \ldots, m-1$ and $x_{i} \rightarrow x_{i}^{-1}$ for $i=m, \ldots, n$, in (2.3), we get

$$
Z_{\mathbb{K}}^{(N)}\left(\boldsymbol{s}, \chi ; \widetilde{\Theta}_{J}, J\right)=\int_{\mathbb{K}^{N}} \widetilde{\Theta}_{J}(\boldsymbol{x}) \frac{F_{J}(x, \boldsymbol{s}, \boldsymbol{\chi})}{\prod_{i=m}^{n}\left|x_{i}\right|_{\mathbb{K}}^{2}} \prod_{i=1}^{N} d x_{i},
$$

where

$$
\begin{aligned}
F_{J}(x, \boldsymbol{s}, \boldsymbol{\chi}):= & \prod_{\substack{l \leq i \leq m-1 \\
m \leq j \leq n}}\left\{\left|x_{i} x_{j}-1\right|_{\mathbb{K}}^{s_{i j}} \chi_{i j}\left(x_{i} x_{j}-1\right)\left|x_{j}\right|_{\mathbb{K}}^{-s_{i j}} \chi_{i j}^{-1}\left(x_{j}\right)\right\} \times \\
& \prod_{\substack{l \leq i<j \leq m-1\\
}}\left\{\left|x_{i}-x_{j}\right|_{\mathbb{K}}^{s_{i j}} \chi_{i j}\left(x_{i}-x_{j}\right)\right\} \times \\
& \prod_{m \leq i<j \leq n}\left\{\left|x_{i}-x_{j}\right|_{\mathbb{K}}^{s_{i j}} \chi_{i j}\left(x_{i}-x_{j}\right)\left|x_{i}\right|_{\mathbb{K}}^{-s_{i j}} \chi_{i j}^{-1}\left(x_{i}\right)\left|x_{j}\right|_{\mathbb{K}}^{-s_{i j}} \chi_{i j}^{-1}\left(x_{j}\right)\right\} .
\end{aligned}
$$

By using Hironaka's resolution of singularities theorem, one establishes the existence of meromorphic continuations (regularizations) for each integral $Z_{\mathbb{K}}^{(N)}\left(s, \chi ; \widetilde{\Theta}_{J}, J\right)$, see e.g. [46]. Here we can show the existence of a common domain where all these integrals converge. In the cases $\mathbb{K}=\mathbb{R}, \mathbb{Q}_{p}$, the description of the possible poles for the integrals $Z_{\mathbb{K}}^{(N)}\left(s, \chi ; \widetilde{\Theta}_{J}, J\right)$ does not depend on $\chi$, see ([38], Theorems 5.4.1, 8.2.1). Consequently, all the results presented in sections 5 and 6 , including Theorem 6.1, extend to these integrals, by replacing $N$ by $N+3$.

For an arbitrary local field $\mathbb{K}$ of characteristic zero, by considering

$$
\left|Z_{\mathbb{K}}^{(N)}(s, \chi)\right|,\left|Z_{\mathbb{K}}^{(N)}\left(s, \chi ; \widetilde{\Theta}_{J}, J\right)\right|,
$$


and using Theorem 4.1, we conclude that all these integrals converge (and are holomorphic) in an open domain, containing the set

$$
-\frac{2}{N+1}<\operatorname{Re}\left(s_{i j}\right)<-\frac{2}{N+3} \quad \text { for all } i j .
$$

And consequently $\left|Z_{\mathbb{K}}^{(N)}\left(s, \widetilde{s}, \operatorname{sgn}_{\mathbb{K}}\right)\right|$ also converges in this neighborhood.

Finally, we regularize $A_{\mathbb{K}}^{(N)}\left(\boldsymbol{k}, \theta, \operatorname{sgn}_{\mathbb{K}}\right)$ by taking

$$
A_{\mathbb{K}}^{(N)}\left(\boldsymbol{k}, \theta, \operatorname{sgn}_{\mathbb{K}}\right)=\left.Z_{\mathbb{K}}^{(N)}\left(\boldsymbol{s}, \widetilde{\boldsymbol{s}}, \operatorname{sgn}_{\mathbb{K}}\right)\right|_{s_{i j}=\boldsymbol{k}_{i} \boldsymbol{k}_{j}, \widetilde{s_{i j}}=\boldsymbol{k}_{i} \theta \boldsymbol{k}_{j}} .
$$

The fact that $A_{\mathbb{K}}^{(N)}\left(\boldsymbol{k}, \theta, \operatorname{sgn}_{\mathbb{K}}\right)$ admits an extension which is meromorphic in the kinematic parameters is established as in the proof of Theorem 7.1.

\subsection{Koba-Nielsen local zeta functions and $1 D$ log-Coulomb gases}

The Koba-Nielsen local zeta functions are partition functions of certain $1 D$ log-Coulomb gases. It is worth to mention that a similar connection has been reported in conformal field theory, see e.g. [41, 42], see also [6] and the references therein. Let $\left(\mathbb{K},|\cdot|_{\mathbb{K}}\right)$ be a local field of characteristic zero. The sites of a configuration of a log Koba-Nielsen-Coulomb gas are vectors of the form $\left(0, x_{2}, \ldots, x_{N-2}, 1\right) \in \mathbb{K}^{N-1}$, which means that two sites are fixed: $x_{1}=0$ and $x_{N-1}=1$. At the site $x_{i}$ there is a charge $e_{i} \in \mathbb{R}$. We set $\boldsymbol{e}=\left(e_{i}\right)_{1 \leq i \leq N-1}$. The Hamiltonian of the log Koba-Nielsen-Coulomb gas is

$$
\begin{aligned}
H_{\mathbb{K}}\left(x_{2}, \ldots, x_{N-2} ; \boldsymbol{e}\right)= & -\sum_{i=2}^{N-2} \ln \left|x_{i}\right|_{\mathbb{K}}^{e_{i} e_{1}}-\sum_{i=2}^{N-2} \ln \left|1-x_{i}\right|_{\mathbb{K}}^{e_{i} e_{(N-1) i}} \\
& -\sum_{2 \leq i<j \leq N-2} \ln \left|x_{i}-x_{j}\right|_{\mathbb{K}}^{e_{i} e_{j}} .
\end{aligned}
$$

The statistical mechanics of this Coulomb gas is described by the Gibbs measure

$$
\frac{e^{-\beta H_{\mathbb{K}}\left(x_{2}, \ldots, x_{N-2} ; e\right)}}{\mathcal{Z}_{\mathbb{K}, N, \boldsymbol{e}}(\beta)} \prod_{i=2}^{N-2} d x_{i}
$$

where $\prod_{i=2}^{N-2} d x_{i}$ is a normalized Haar measure of the topological group $\left(\mathbb{K}^{N-3},+\right), \beta>0$ is the inverse temperature and $\mathcal{Z}_{\mathbb{K}, \beta, N, \boldsymbol{e}}$ is a normalization constant, the partition function, defined as

$$
\mathcal{Z}_{\mathbb{K}, N, \boldsymbol{e}}(\beta)=\int_{\mathbb{K}^{N-3}} \prod_{i=2}^{N-2}\left|x_{i}\right|_{\mathbb{K}}^{\beta e_{i} e_{1}}\left|1-x_{i}\right|_{\mathbb{K}}^{\beta e_{i} e_{(N-1) i}} \prod_{2 \leq i<j \leq N-2}\left|x_{i}-x_{j}\right|_{\mathbb{K}}^{\beta e_{i} e_{j}} \prod_{i=2}^{N-2} d x_{i}
$$

Then, Theorem 6.1 implies that $\mathcal{Z}_{\mathbb{K}, N, \boldsymbol{e}}(\beta)$ converges in some region determined by the $\beta e_{i} e_{j}$, and it admits a meromorphic continuation to the whole $\mathbb{C}^{\frac{N(N-3)}{2}}$. It is interesting to mention that positive poles of $\mathcal{Z}_{\mathbb{K}, N, \boldsymbol{e}}(\beta)$ are related with phase transitions, see [61] and the references therein. 


\section{Multivariate local zeta functions and embedded resolution of singular- ities}

Let $f_{1}(x), \ldots, f_{m}(x) \in \mathbb{R}\left[x_{1}, \ldots, x_{n}\right]$ be non-constant polynomials; we denote by $D:=$ $\cup_{i=1}^{m} f_{i}^{-1}(0)$ the divisor attached to them. We set $\boldsymbol{f}:=\left(f_{1}, \ldots, f_{m}\right)$ and $\boldsymbol{s}:=\left(s_{1}, \ldots, s_{m}\right) \in$ $\mathbb{C}^{m}$. For each $\Theta: \mathbb{R}^{n} \rightarrow \mathbb{C}$ smooth with compact support, the multivariate local zeta function attached to $(\boldsymbol{f}, \Theta)$ is defined as

$$
Z_{\Theta}(\boldsymbol{f}, \boldsymbol{s}):=\int_{\mathbb{R}^{n} \backslash D} \Theta(x) \prod_{i=1}^{m}\left|f_{i}(x)\right|^{s_{i}} \prod_{i=1}^{n} d x_{i},
$$

when $\operatorname{Re}\left(s_{i}\right)>0$ for all $i$. Integrals of type (3.1) are analytic functions, and they admit meromorphic continuations to the whole $\mathbb{C}^{m}$, see $[37,38,46]$. By applying Hironaka's resolution of singularities theorem to $D$, the study of integrals of type (3.1) is reduced to the case of monomials integrals, which can be studied directly, see e.g. [37, 38, 46].

Theorem 3.1 (Hironaka, [35]). There exists an embedded resolution $\sigma: X \rightarrow \mathbb{R}^{n}$ of $D$, that is,

(i) $X$ is an $n$-dimensional $\mathbb{R}$-analytic manifold, $\sigma$ is a proper $\mathbb{R}$-analytic map which is a composition of a finite number of blow-ups at closed submanifolds, and which is an isomorphism outside of $\sigma^{-1}(D)$;

(ii) $\sigma^{-1}(D)$ is a normal crossings divisor, meaning that $\sigma^{-1}(D)=\cup_{i \in T} E_{i}$, where the $E_{i}$ are closed submanifolds of $X$ of codimension one, each equipped with an m-tuple of nonnegative integers $\left(N_{f_{1}, i}, \ldots, N_{f_{m}, i}\right)$ and a positive integer $v_{i}$, satisfying the following. At every point $b$ of $X$ there exist local coordinates $\left(y_{1}, \ldots, y_{n}\right)$ on $X$ around $b$ such that, if $E_{1}, \ldots, E_{r}$ are the $E_{i}$ containing $b$, we have on some open neighborhood $V$ of $b$ that $E_{i}$ is given by $y_{i}=0$ for $i \in\{1, \ldots, r\}$,

$$
\sigma^{*}\left(d x_{1} \wedge \ldots \wedge d x_{n}\right)=\eta(y)\left(\prod_{i=1}^{r} y_{i}^{v_{i}-1}\right) d y_{1} \wedge \ldots \wedge d y_{n},
$$

and

$$
f_{j}^{*}(y):=\left(f_{j} \circ \sigma\right)(y)=\varepsilon_{f_{j}}(y) \prod_{i=1}^{r} y_{i}^{N_{f_{j}, i}} \quad \text { for } j=1, \ldots, m,
$$

where $\eta(y)$ and the $\varepsilon_{f_{j}}(y)$ belong to $\mathcal{O}_{X, b}^{\times}$, the group of units of the local ring of $X$ at $b$.

There are two kinds of submanifolds $E_{i}, i \in T$. Each blow-up creates an exceptional variety $E_{i}$, the image by $\sigma$ of any of these $E_{i}$ has codimension at least two in $\mathbb{R}^{n}$. The other $E_{i}$ are the so-called strict transforms of the irreducible components of $D$.

When using Hironaka's resolution theorem, we will identify the Lebesgue measure $\prod_{i=1}^{n} d x_{i}$ with the measure induced by the top differential form $d x_{1} \wedge \ldots \wedge d x_{n}$ in $\mathbb{R}^{n}$. For a discussion on the basic aspects of analytic manifolds and resolution of singularities, the reader may consult ([38], chapter 2). More generally, Hironaka's resolution theorem is valid over any field of characteristic zero, in particular over the local fields $\mathbb{R}, \mathbb{C}$, the field of $p$-adic numbers $\mathbb{Q}_{p}$, or a finite extension of $\mathbb{Q}_{p}$. 
The resulting monomial integrals are then handled by the following lemma, which is an easy variation of well-known results, see e.g. ([4], chapter II, section 7, Lemme 4), ([25], Lemme 3.1), ([31], chapter I, section 3.2), and ([37], Lemma 4.5).

Lemma 3.1. Consider the integral

$$
J\left(s_{1}, \ldots, s_{m}\right)=\int_{\mathbb{R}^{n}} \Phi\left(y, s_{1}, \ldots, s_{m}\right) \prod_{i=1}^{r}\left|y_{i}\right|^{\sum_{j=1}^{m} a_{j, i} s_{j}+b_{i}-1} \prod_{i=1}^{n} d y_{i},
$$

where $1 \leq r \leq n$, for each $i$ the $a_{j, i}$ are integers (not all zero) and $b_{i}$ is an integer, and $\Phi\left(y, s_{1}, \ldots, s_{m}\right)$ is a smooth function with support in the polydisc

$$
\left\{y \in \mathbb{R}^{n} ;\left|y_{i}\right|<1, \text { for } i=1, \ldots, n\right\},
$$

which is holomorphic in $s_{1}, \ldots, s_{m}$. Set

$$
\mathcal{R}:=\bigcap_{i \in\{1, \ldots, r\}}\left\{\left(s_{1}, \ldots, s_{m}\right) \in \mathbb{C}^{m} ; \sum_{j=1}^{m} a_{j, i} \operatorname{Re}\left(s_{j}\right)+b_{i}>0\right\} .
$$

Then the following assertions hold:

(i) if all the $a_{j, i}$ are nonnegative integers (not all zero) and $b_{i}$ is a positive integer, then $\mathcal{R} \neq \emptyset$. More precisely, $\left\{\left(s_{1}, \ldots, s_{m}\right) \in \mathbb{C}^{m} ; \operatorname{Re}\left(s_{j}\right)>0, j=1, \cdots, m\right\} \subset \mathcal{R} ;$

(ii) if $\mathcal{R} \neq \emptyset$, then $J\left(s_{1}, \ldots, s_{m}\right)$ is convergent and defines a holomorphic function in the domain $\mathcal{R}$;

(iii) if $\mathcal{R} \neq \emptyset, J\left(s_{1}, \ldots, s_{m}\right)$ admits an analytic continuation to the whole $\mathbb{C}^{m}$, as a meromorphic function with poles belonging to

$$
\bigcup_{1 \leq i \leq r} \bigcup_{t \in \mathbb{N}}\left\{\sum_{j=1}^{m} a_{j, i} s_{j}+b_{i}+t=0\right\} .
$$

Remark 1. Integrals of the form $J\left(s_{1}, \ldots, s_{m}\right)$, where the $a_{j, i}$ and $b_{i}$ are positive integers, have been extensively studied, see e.g. ([4], chapter II, § 7, Lemme 4), ([25], Lemme 3.1), ([31], chapter I, section 3.2), and ([37], Lemma 4.5).

Combining Theorem 3.1 and Lemma 3.1, the precise conclusion is as follows.

Theorem 3.2. Let $f_{1}(x), \ldots, f_{m}(x) \in \mathbb{R}\left[x_{1}, \ldots, x_{n}\right]$ be non-constant polynomials and $\Theta$ : $\mathbb{R}^{n} \rightarrow \mathbb{C}$ a smooth function with compact support, to which we associate the multivariate local zeta function $Z_{\Theta}(\boldsymbol{f}, \boldsymbol{s})$ as in (3.1). Fix an embedded resolution $\sigma: X \rightarrow \mathbb{R}$ of $D=$ $\cup_{i=1}^{m} f_{i}^{-1}(0)$ as in Theorem 3.1. Then

(i) $Z_{\Theta}(\boldsymbol{f}, \boldsymbol{s})$ is convergent and defines a holomorphic function in the region

$$
\sum_{j=1}^{m} N_{f_{j}, i} \operatorname{Re}\left(s_{j}\right)+v_{i}>0, \quad \text { for } i \in T
$$

(ii) $Z_{\Theta}(\boldsymbol{f}, \boldsymbol{s})$ admits a meromorphic continuation to the whole $\mathbb{C}^{m}$, with poles belonging to

$$
\bigcup_{i \in T} \bigcup_{t \in \mathbb{N}}\left\{\sum_{j=1}^{m} N_{f_{j}, i} s_{j}+v_{i}+t=0\right\} .
$$


(iii) In particular, in the case of one polynomial $f$, i.e., $m=1$, the pair $\left(N_{f, i}, v_{i}\right)$ is called the numerical datum of $E_{i}$, and the set $\left\{\left(N_{f, i}, v_{i}\right) ; i \in T\right\}$ is called the numerical data of the resolution $\sigma$. It is well known that $\min _{i \in T} \frac{v_{i}}{N_{f, i}}$ does not depend on the choice of the resolution $\sigma$ (this value is called the real log canonical threshold of $f$ ). Furthermore, $Z_{\Theta}(f, s)$ is holomorphic in the half-space $\operatorname{Re}(s)>-\min _{i \in T} \frac{v_{i}}{N_{f, i}}$, and the possible poles of its meromorphic continuation belong to the set $\cup_{i \in T}\left(-\frac{v_{i}+\mathbb{N}}{N_{f, i}}\right)$.

The above theorem is a consequence of the work of many people: Gel'fand (I. M. and S. I.), Bernstein, Atiyah, Igusa, Loeser, as far as we know. We will use this result, as well as Lemma 3.1, along this article; the formulation that we are giving here is the one we require. The formulation of Lemma 3.1 will be crucial for dealing with certain non-classical local zeta functions that occur in section 5.4.

\section{Local zeta functions of Koba-Nielsen type}

We consider $\mathbb{R}^{N-3}$ as an $\mathbb{R}$-analytic manifold, with $N \geq 4$, and use $\left\{x_{2}, \ldots, x_{N-2}\right\}$ as a coordinate system. In addition, we take

$$
D_{N}:=\left\{x \in \mathbb{R}^{N-3} ; \prod_{i=2}^{N-2} x_{i} \prod_{i=2}^{N-2}\left(1-x_{i}\right) \prod_{2 \leq i<j \leq N-2}\left(x_{i}-x_{j}\right)=0\right\},
$$

and use $\prod_{i=2}^{N-2} d x_{i}$ to denote the measure induced by the top differential form $d x_{2} \wedge \ldots \wedge$ $d x_{N-2}$.

Definition 1. A Koba-Nielsen local zeta function is defined to be an integral of the form

$$
Z^{(N)}(s):=\int_{\mathbb{R}^{N-3} \backslash D_{N}} \prod_{j=2}^{N-2}\left|x_{j}\right|^{s_{1 j}} \prod_{j=2}^{N-2}\left|1-x_{j}\right|^{s_{(N-1) j}} \prod_{2 \leq i<j \leq N-2}\left|x_{i}-x_{j}\right|^{s_{i j}} \prod_{i=2}^{N-2} d x_{i},
$$

where $\boldsymbol{s}=\left(s_{i j}\right)=\cup_{j=2}^{N-2}\left\{s_{1 j}, s_{(N-1) j}\right\} \cup \cup_{2 \leq i<j \leq N-2}\left\{s_{i j}\right\}$ is a list consisting of $\mathbf{d}$ complex variables, where

$$
\mathbf{d}:=\left\{\begin{array}{l}
2(N-3)+\left(\begin{array}{c}
N-3 \\
2
\end{array}\right) \text { if } N \geq 5=\frac{N(N-3)}{2} . \\
\text { if } N=4
\end{array}\right.
$$

For later use in formulas, it will be convenient to put also $s_{i j}=s_{j i}$ for any occurring $\{i, j\}$. For simplicity of notation, we will put $\mathbb{R}^{N-3}$ instead of $\mathbb{R}^{N-3} \backslash D_{N}$ in (4.2), and similarly in other such integrals.

In order to regularize the integral (4.2), we will use a partition of $\mathbb{R}^{N-3}$ constructed using a smooth function $\chi: \mathbb{R} \rightarrow \mathbb{R}$ satisfying

$$
\chi(x)=\left\{\begin{array}{l}
1 \text { if } \quad x \in[-2,2] \\
0 \text { if } x \in(-\infty,-2-\epsilon] \cup[2+\epsilon,+\infty),
\end{array}\right.
$$


for some fixed positive $\epsilon$ sufficiently small. The existence of such a function is well-known, see e.g. ([36], section 1.4), ([38], section 5.2). Let us mention that the number 2 was chosen in an arbitrary form, the key point is that the interval $[0,1]$ is included in the locus where $\chi \equiv 1$.

Definition 2. For $I \subseteq\{2, \ldots, N-2\}$, including the empty set, we set

$$
\varphi_{I}: \mathbb{R}^{N-3} \rightarrow \mathbb{R}: x \mapsto \prod_{i \in I} \chi\left(x_{i}\right) \prod_{i \notin I}\left(1-\chi\left(x_{i}\right)\right),
$$

with the convention that $\prod_{i \in \emptyset} \equiv 1$.

Then $\varphi_{I} \in C^{\infty}\left(\mathbb{R}^{N-3}\right)$ and $\sum_{I} \varphi_{I}(x) \equiv 1$, for $x \in \mathbb{R}^{N-3}$. By using this partition of the unity, we have

$$
Z^{(N)}(s)=\sum_{I} Z_{I}^{(N)}(s)
$$

with

$$
Z_{I}^{(N)}(\boldsymbol{s}):=\int_{\mathbb{R}^{N-3}} \varphi_{I}(x) \prod_{j=2}^{N-2}\left|x_{j}\right|^{s_{1 j}} \prod_{j=2}^{N-2}\left|1-x_{j}\right|^{s_{(N-1) j}} \prod_{2 \leq i<j \leq N-2}\left|x_{i}-x_{j}\right|^{s_{i j}} \prod_{i=2}^{N-2} d x_{i} .
$$

In the case $I=\{2, \ldots, N-2\}, Z_{I}^{(N)}(\boldsymbol{s})$ is a classical multivariate Igusa local zeta function (since then $\varphi_{I}(x)$ has compact support). These integrals are holomorphic functions in a region including $\operatorname{Re}\left(s_{i j}\right)>0$ for all $i j$, and they admit meromorphic continuations to the whole $\mathbb{C}^{\mathbf{d}}$, see Theorem 3.2.

In the case $I \neq\{2, \ldots, N-2\}$, by changing variables in (4.7) as $x_{i} \rightarrow \frac{1}{x_{i}}$ for $i \notin I$, and $x_{i} \rightarrow x_{i}$ for $i \in I$, we have $\prod_{i=2}^{N-2} d x_{i} \rightarrow \prod_{i \notin I} \frac{1}{\left|x_{i}\right|^{2}} \prod_{i=2}^{N-2} d x_{i}$, and by setting $\tilde{\chi}\left(x_{i}\right):=$ $1-\chi\left(\frac{1}{x_{i}}\right)$ for $i \notin I$, i.e.,

$$
\tilde{\chi}\left(x_{i}\right)=\left\{\begin{array}{l}
1 \text { if }\left|x_{i}\right| \leq \frac{1}{2+\epsilon} \\
0 \text { if }\left|x_{i}\right| \geq \frac{1}{2},
\end{array},\right.
$$

we have that supp $\tilde{\chi} \subseteq\left[-\frac{1}{2}, \frac{1}{2}\right]$ and $\tilde{\chi} \in C^{\infty}(\mathbb{R})$. Now setting $\widetilde{\varphi}_{I}(x):=\prod_{i \notin I} \tilde{\chi}\left(x_{i}\right)$ $\prod_{i \in I} \chi\left(x_{i}\right)$, and

$$
\begin{aligned}
F_{I}(x, s):= & \prod_{j \in I}\left|x_{j}\right|^{s_{1 j}} \prod_{j=2}^{N-2}\left|1-x_{j}\right|^{s_{(N-1) j}} \prod_{\substack{2 \leq i<j \leq N-2 \\
i, j \in I}}\left|x_{i}-x_{j}\right|^{s_{i j}} \times \\
& \prod_{\substack{i \leq i<j \leq N-2 \\
i, j \notin I}}\left|x_{i}-x_{j}\right|^{s_{i j}} \prod_{\substack{2 \leq i<j \leq N-2 \\
i \notin I, j \in I}}\left|1-x_{i} x_{j}\right|^{s_{i j}} \prod_{\substack{2 \leq i<j \leq N-2 \\
i \in I, j \notin I}}\left|1-x_{i} x_{j}\right|^{s_{i j}},
\end{aligned}
$$

we have

$$
Z_{I}^{(N)}(s)=\int_{\mathbb{R}^{N-3} \backslash D_{I}} \frac{\widetilde{\varphi}_{I}(x) F_{I}(x, s)}{\prod_{i \notin I}\left|x_{i}\right|} s_{\substack{1 i+s_{(N-1) i}+\sum_{2 \leq j \leq N-2} s_{i j}+2 \\ j \neq i}} \prod_{i=2}^{N-2} d x_{i}
$$


where $D_{I}$ is the divisor defined by the polynomial

$$
\begin{gathered}
\prod_{i=2}^{N-2} x_{i} \prod_{i=2}^{N-2}\left(1-x_{i}\right) \prod_{\substack{2 \leq i<j \leq N-2 \\
i, j \in I}}\left(x_{i}-x_{j}\right) \prod_{\substack{2 \leq i<j \leq N-2 \\
i, j \notin I}}\left(x_{i}-x_{j}\right) \times \\
\prod_{\substack{i<j \leq N-2 \\
i \notin I, j \in I}}\left(1-x_{i} x_{j}\right) \prod_{\substack{2 \leq i<j \leq N-2 \\
i \in I, j \notin I}}\left(1-x_{i} x_{j}\right) .
\end{gathered}
$$

From the expression in (4.8), it is not clear at all whether integrals of type $Z_{I}^{(N)}(s)$, with $I \neq$ $\{2, \ldots, N-2\}$, converge for some value of $s$. These integrals are not classical multivariate local zeta functions, and Theorem 3.2 does not apply to them. We will show that they define holomorphic functions on some nonempty open in $\mathbb{C}^{\mathbf{d}}$, and admit meromorphic continuations to the whole $\mathbb{C}^{\mathbf{d}}$. To establish this result we will use Lemma 3.1 and embedded resolution of singularities. The technical statement is as follows.

Lemma 4.1. For any $I \subseteq\{2, \ldots, N-2\}$, the function $Z_{I}^{(N)}(s)$ is holomorphic in $s$ on the solution set $\mathcal{H}(I)$ of a system of inequalities of the form

$$
\mathcal{H}(I):=\left\{s_{i j} \in \mathbb{C}^{\mathbf{d}} ; \quad \sum_{i j \in M(I)} N_{i j, k}(I) \operatorname{Re}\left(s_{i j}\right)+\gamma_{k}(I)>0, \text { for } k \in T(I)\right\} \neq \emptyset,
$$

where $N_{i j, k}(I), \gamma_{k}(I) \in \mathbb{Z}$, and $M(I), T(I)$ are finite sets. More precisely, for each $k$, either all numbers $N_{i j, k}(I)$ are equal to 0 or 1 and $\gamma_{k}(I)>0$, or all numbers $N_{i j, k}(I)$ are equal to 0 or -1 and $\gamma_{k}(I)<0$.

In addition, $Z_{I}^{(N)}(s)$ admits an analytic continuation to the whole $\mathbb{C}^{\mathbf{d}}$, as a meromorphic function with poles belonging to

$$
\mathcal{P}(I):=\bigcup_{t \in \mathbb{N}} \bigcup_{k \in T(I)}\left\{s_{i j} \in \mathbb{C}^{\mathbf{d}} ; \sum_{i j \in M(I)} N_{i j, k}(I) s_{i j}+\gamma_{k}(I)+t=0\right\} .
$$

Proof. By applying Hironaka's Theorem 3.1 to the divisors $D_{N}$ or $D_{I}$, and by using a suitable partition of the unity, each integral $Z_{I}^{(N)}(s)$ becomes a finite sum of monomial type integrals. The important statement here is that $\mathcal{H}(I) \neq \emptyset$ if $I \neq\{2, \ldots, N-2\}$; this is shown later in Propositions 5.1, 5.2, together with the specifications concerning the numbers $N_{i j, k}(I)$ and $\gamma_{k}(I)$. Then the meromorphic continuation of the integrals follows from Lemma 3.1.

Then, by formula (4.6), the integral $Z^{(N)}(s)$ will be a finite sum of functions $Z_{I}^{(N)}(s)$, holomorphic on the domain $\mathcal{H}(I)$ in $\mathbb{C}^{\mathbf{d}}$, that however depends on $I$. Hence, the convergence and the analytic continuation of the integral $Z^{(N)}(s)$ is not a direct consequence of the existence of meromorphic continuations for the integrals $Z_{I}^{(N)}(s)$. We will show that all the integrals $Z_{I}^{(N)}(s)$ are holomorphic in a common domain, and then formula (4.6) allows us to construct a meromorphic continuation of $Z^{(N)}(s)$.

More precisely, in order to show that $\cap_{I} \mathcal{H}(I)$ contains a non-empty open subset of $\mathbb{C}^{\mathbf{d}}$, we will take $\operatorname{Re}\left(s_{i j}\right)=\operatorname{Re}(s)$ for any $i j$ and any $I$, and show that the solution set (of the 
system of inequalities obtained in this way) contains a non-empty open subset of $\mathbb{C}$. This fact will be established by studying possible poles of functions of the form

$$
Z_{I}^{(N)}(s):=\left.Z_{I}^{(N)}(s)\right|_{s_{i j}=s}
$$

and proving that $Z^{(N)}(s):=\left.Z^{(N)}(s)\right|_{s_{i j}=s}$ is a holomorphic function in the region

$$
-\frac{2}{N-2}<\operatorname{Re}(s)<-\frac{2}{N}
$$

see again Propositions 5.1, 5.2. A somewhat more elaborate argument yields the following more precise result.

Theorem 4.1. The Koba-Nielsen local zeta function $Z^{(N)}(s)$ is a holomorphic function in the solution set $\cap_{I} \mathcal{H}(I)$, see (4.9), in $\mathbb{C}^{\mathbf{d}}$, which contains the set

$$
-\frac{2}{N-2}<\operatorname{Re}\left(s_{i j}\right)<-\frac{2}{N} \quad \text { for all } i j .
$$

Furthermore, it has a meromorphic continuation, denoted again as $Z^{(N)}(s)$, to the whole $\mathbb{C}^{\mathrm{d}}$, with poles belonging to $\cup_{I} \mathcal{P}(I)$, see (4.10).

The meromorphic continuation of $Z^{(N)}(s)$ does not depend on the choice of the function $\chi$. Suppose that we pick another smooth function $\chi_{0}$, with compact support, such that $[-1,1] \subset \operatorname{supp} \chi_{0}$ and $\left.\chi_{0}\right|_{[-1,1]} \equiv 1$. Then $Z^{(N)}(s)$ has a meromorphic continuation, denoted now as $Z_{0}^{(N)}(s)$, to the whole $\mathbb{C}^{\mathbf{d}}$ minus a countable number of hyperplanes. Both $Z^{(N)}(s)$ and $Z_{0}^{(N)}(s)$ are holomorphic in $\mathbb{C}^{\mathbf{d}}$ minus a countable number of hyperplanes and coincide in an open set of $\mathbb{C}^{\mathbf{d}}$, where both functions are holomorphic. Consequently, by the analytic continuation principle, $Z^{(N)}(s)=Z_{0}^{(N)}(s)$ in $\mathbb{C}^{\mathbf{d}}$ minus a countable number of hyperplanes.

\section{$5 \quad$ Road map of the proof}

Since the $D_{I}$ are so-called hyperplane arrangements (their irreducible components are all hyperplanes), there is a well-known and straightforward way to construct an embedded resolution for them. In fact, we only have to modify the locus where the defining equation of $D_{I}$ is not locally monomial and that moreover is contained in the support of $\varphi_{I}$ or $\widetilde{\varphi}_{I}$. The standard algorithm is to blow up consecutively in relevant centres of increasing dimension contained in that locus, until the total inverse image of $D_{I}$ becomes a normal crossings divisor, i.e., its defining equation becomes locally monomial. Then we conclude by repeated applications of Lemma 3.1.

For readers who are not familiar with these notions, we will treat explicitly the first blow-ups of such a resolution, presented as explicit change of variables operations, simplifying the original integrals $Z_{I}^{(N)}(s)$.

First, in order to get a feeling for the method, we present the easy case $N=4$ and especially the case $N=5$, where we explain in detail the set $\mathcal{H}(I)$ of Lemma 4.1. 


\subsection{Example}

Fix $N=4$. Then $Z^{(4)}(s)=Z_{\{2\}}^{(4)}(s)+Z_{\emptyset}^{(4)}(s)$. We have that

$$
Z_{\{2\}}^{(4)}(s)=\int_{\mathbb{R}} \chi\left(x_{2}\right)\left|x_{2}\right|^{s_{12}}\left|1-x_{2}\right|^{s_{32}} d x_{2},
$$

which converges and is analytic when $\operatorname{Re}\left(s_{12}\right)+1>0$ and $\operatorname{Re}\left(s_{32}\right)+1>0$, and

$$
Z_{\emptyset}^{(4)}(\boldsymbol{s})=\int_{\mathbb{R}} \tilde{\chi}\left(x_{2}\right)\left|x_{2}\right|^{-s_{12}-s_{32}-2}\left|1-x_{2}\right|^{s_{32}} d x_{2},
$$

which converges and is analytic when $-\operatorname{Re}\left(s_{12}\right)-\operatorname{Re}\left(s_{32}\right)-1>0$ and $\operatorname{Re}\left(s_{32}\right)+1>0$. We conclude by Lemma 3.1 that $Z^{(4)}(s)$ converges and is analytic in the region

$$
\operatorname{Re}\left(s_{12}\right)>-1, \quad \operatorname{Re}\left(s_{32}\right)>-1, \quad \operatorname{Re}\left(s_{12}\right)+\operatorname{Re}\left(s_{32}\right)<-1 .
$$

\subsection{Example}

Fix $N=5$. Then $Z^{(5)}(\boldsymbol{s})=Z_{\{2,3\}}^{(5)}(\boldsymbol{s})+Z_{\{3\}}^{(5)}(\boldsymbol{s})+Z_{\{2\}}^{(5)}(\boldsymbol{s})+Z_{\emptyset}^{(5)}(\boldsymbol{s})$. We start with

$$
Z_{\{2,3\}}^{(5)}(s)=\int_{\mathbb{R}^{2}} \chi\left(x_{2}\right) \chi\left(x_{3}\right)\left|x_{2}\right|^{s_{12}}\left|x_{3}\right|^{s_{13}}\left|1-x_{2}\right|^{s_{42}}\left|1-x_{3}\right|^{s_{43}}\left|x_{2}-x_{3}\right|^{s_{23}} d x_{2} d x_{3} .
$$

The arrangement $D_{5}$, given by $x_{2} x_{3}\left(1-x_{2}\right)\left(1-x_{3}\right)\left(x_{2}-x_{3}\right)=0$, is not locally monomial only at the points $(0,0)$ and $(1,1)$. We pick a partition of the unity, $\sum_{i=0}^{2} \Omega_{i}\left(x_{2}, x_{3}\right)=1$, where $\Omega_{0}$ and $\Omega_{1}$ are smooth functions with support in a small neighborhood of $(0,0)$ and $(1,1)$, respectively. This reduces the computation to neighborhoods of each of these two points. That is, we can write $Z_{\{2,3\}}^{(5)}(s)=\sum_{j=0}^{2} Z_{\Omega_{j}}^{(5)}(s)$, where the $Z_{\Omega_{j}}^{(5)}(s)$ are integrals as in (5.1), replacing $\chi\left(x_{2}\right) \chi\left(x_{3}\right)$ by $\Omega_{j}\left(x_{2}, x_{3}\right)$, and the relevant integrals to compute are $Z_{\Omega_{0}}^{(5)}(s)$ and $Z_{\Omega_{1}}^{(5)}(s)$.

Around $(0,0)$, the factor $\left|1-x_{2}\right|^{s_{42}}\left|1-x_{3}\right|^{s_{43}}$ can be neglected from the point of view of convergence and holomorphy, hence here we only need an embedded resolution of $x_{2} x_{3}\left(x_{2}-x_{3}\right)=0$, which is obtained by a blow-up at the origin. This just means the two changes of variables

$$
\begin{array}{rlrl}
\sigma_{0}: \mathbb{R}^{2} \rightarrow \mathbb{R}^{2}: u_{2} & \mapsto x_{2} & =u_{2} & \text { and } \\
u_{3} & \mapsto x_{3}=u_{2} u_{3}: \mathbb{R}^{2} \rightarrow \mathbb{R}^{2}: u_{2} & \mapsto x_{2}=u_{2} u_{3} \\
u_{3} & \mapsto x_{3}=u_{3} .
\end{array}
$$

By symmetry it is enough to consider the first one, yielding as contribution to $Z_{\Omega_{0}}^{(5)}(s)$ the integral

$$
\int_{\mathbb{R}^{2}}\left(\Omega_{0} \circ \sigma_{0}\right)\left(u_{2}, u_{3}\right)\left|u_{2}\right|^{s_{12}+s_{13}+s_{23}+1}\left|u_{3}\right|^{s_{13}}\left|1-u_{3}\right|^{s_{23}} g(u, s) d u_{2} d u_{3},
$$

where $g(u, s)$ is invertible on the support of $\Omega_{0} \circ \sigma_{0}$ (and can thus be neglected from the point of view of convergence and holomorphy). Hence, we are in the locally monomial setting, and Lemma 3.1 yields the convergence conditions

$$
\operatorname{Re}\left(s_{12}\right)+\operatorname{Re}\left(s_{13}\right)+\operatorname{Re}\left(s_{23}\right)+2>0, \quad \operatorname{Re}\left(s_{23}\right)+1>0, \quad \operatorname{Re}\left(s_{13}\right)+1>0 .
$$


The other chart of the blow-up, i.e., the change of variables $\sigma_{0}^{\prime}$, yields the same first and second condition and also

$$
\operatorname{Re}\left(s_{12}\right)+1>0
$$

Completely similarly, for the convergence of $Z_{\Omega_{1}}^{(5)}(s)$, we need also the new conditions

$$
\operatorname{Re}\left(s_{42}\right)+\operatorname{Re}\left(s_{43}\right)+\operatorname{Re}\left(s_{23}\right)+2>0, \quad \operatorname{Re}\left(s_{42}\right)+1>0, \quad \operatorname{Re}\left(s_{43}\right)+1>0 .
$$

Note that the conditions coming from the locally monomial integral $Z_{\Omega_{2}}^{(5)}(s)$ are already included now. Next,

$$
Z_{\{3\}}^{(5)}(s)=\int_{\mathbb{R}^{2}} \widetilde{\chi}\left(x_{2}\right) \chi\left(x_{3}\right)\left|x_{2}\right|^{-s_{12}-s_{42}-s_{23}-2}\left|x_{3}\right|^{s_{13}}\left|1-x_{2}\right|^{s_{42}}\left|1-x_{3}\right|^{s_{43}}\left|1-x_{2} x_{3}\right|^{s_{23}} d x_{2} d x_{3} .
$$

Since $x_{2} x_{3}\left(1-x_{2}\right)\left(1-x_{3}\right)\left(1-x_{2} x_{3}\right)$ is locally monomial in the support of $\tilde{\chi}\left(x_{2}\right) \chi\left(x_{3}\right)$, the only new condition that arises is

$$
-\operatorname{Re}\left(s_{12}\right)-\operatorname{Re}\left(s_{42}\right)-\operatorname{Re}\left(s_{23}\right)-1>0 .
$$

Completely analogously, $Z_{\{2\}}^{(5)}(s)$ induces the extra condition

$$
-\operatorname{Re}\left(s_{13}\right)-\operatorname{Re}\left(s_{43}\right)-\operatorname{Re}\left(s_{23}\right)-1>0 .
$$

Finally, we must consider

$$
\begin{aligned}
& Z_{\emptyset}^{(5)}(\boldsymbol{s})= \\
& \int_{\mathbb{R}^{2}} \tilde{\chi}\left(x_{2}\right) \widetilde{\chi}\left(x_{3}\right)\left|x_{2}\right|^{-s_{12}-s_{42}-s_{23}-2}\left|x_{3}\right|^{-s_{13}-s_{43}-s_{23}-2}\left|1-x_{2}\right|^{s_{42}}\left|1-x_{3}\right|^{s_{43}}\left|x_{2}-x_{3}\right|^{s_{23}} d x_{2} d x_{3} .
\end{aligned}
$$

Geometrically, we have here the same arrangement as for $Z_{\{2,3\}}^{(5)}(s)$; the differences are the powers of $\left|x_{2}\right|$ and $\left|x_{3}\right|$ and the function $\widetilde{\chi}\left(x_{2}\right) \widetilde{\chi}\left(x_{3}\right)$, that does not contain $(1,1)$ in its support. Hence, the only new condition will arise from the blow-up at the origin, namely

$\left(-\operatorname{Re}\left(s_{12}\right)-\operatorname{Re}\left(s_{42}\right)-\operatorname{Re}\left(s_{23}\right)-2\right)+\left(-\operatorname{Re}\left(s_{13}\right)-\operatorname{Re}\left(s_{43}\right)-\operatorname{Re}\left(s_{23}\right)-2\right)+\operatorname{Re}\left(s_{23}\right)+2>0$,

which simplifies to

$$
-\operatorname{Re}\left(s_{12}\right)-\operatorname{Re}\left(s_{42}\right)-\operatorname{Re}\left(s_{23}\right)-\operatorname{Re}\left(s_{13}\right)-\operatorname{Re}\left(s_{43}\right)-2>0 .
$$

The conclusion is that $Z^{(5)}(s)$ converges and is analytic in the region defined by conditions (5.2)-(5.8), that is, for the five indices $i j$,

$$
\begin{aligned}
& \text { all } \operatorname{Re}\left(s_{i j}\right)>-1, \\
& \operatorname{Re}\left(s_{12}\right)+\operatorname{Re}\left(s_{13}\right)+\operatorname{Re}\left(s_{23}\right)>-2, \quad \operatorname{Re}\left(s_{42}\right)+\operatorname{Re}\left(s_{43}\right)+\operatorname{Re}\left(s_{23}\right)>-2, \\
& \operatorname{Re}\left(s_{12}\right)+\operatorname{Re}\left(s_{42}\right)+\operatorname{Re}\left(s_{23}\right)<-1, \quad \operatorname{Re}\left(s_{13}\right)+\operatorname{Re}\left(s_{43}\right)+\operatorname{Re}\left(s_{23}\right)<-1, \\
& \sum_{i j} \operatorname{Re}\left(s_{i j}\right)<-2 .
\end{aligned}
$$


It is immediate to verify that the simpler domain

$$
-\frac{2}{3}<\operatorname{Re}\left(s_{i j}\right)<-\frac{2}{5} \quad \text { for all } i j
$$

is contained in this region. Then, in particular, $Z^{(5)}(s)$ is analytic in the interval $-\frac{2}{3}<$ $\operatorname{Re}(s)<-\frac{2}{5}$.

We now proceed in general, assuming implicitly that $N \geq 6$. The regions generalizing (5.9) can be determined explicitly for any given $N$, but require long lists of inequalities. They will be stated in Proposition 5.3. To keep the exposition more accessible, we will concentrate on the domain generalizing (5.10) and its specialization to $Z^{(N)}(s)$.

\subsection{Case $I=\{2, \ldots, N-2\}$}

Recall that then $Z_{I}^{(N)}(s)$ and $Z_{I}^{(N)}(s)$ are classical local zeta functions, the last one associated to the polynomial

$$
f_{N}(x)=\prod_{i=2}^{N-2} x_{i} \prod_{i=2}^{N-2}\left(1-x_{i}\right) \prod_{2 \leq i<j \leq N-2}\left(x_{i}-x_{j}\right)
$$

We note first that, for convergence of $Z_{I}^{(N)}(\boldsymbol{s})$, at least the conditions $\operatorname{Re}\left(s_{i j}\right)+1>0$, for all $i j$, are needed, since they will certainly appear as powers of (absolute values of) variables in some monomial integral. Geometrically, these conditions are induced by the strict transforms $E_{i}$ in an embedded resolution of the components of $D_{N}$. ( For convergence of $Z_{I}^{(N)}(s)$, they all induce the same condition $\operatorname{Re}(s)+1>0$.)

Next, concerning $Z_{I}^{(N)}(s)$, we have by Theorem 3.2 (iii) that this function is holomorphic in the half-space

$$
\operatorname{Re}(s)>-\min _{i \in T} \frac{v_{i}}{N_{f_{N}, i}},
$$

where $\left\{\left(N_{f_{N}, i}, v_{i}\right) ; i \in T\right\}$ are the numerical data of an embedded resolution $\sigma$ of $D_{N}$. We will explain how to construct such a resolution and obtain that this minimum value is $\frac{2}{N-2}$. Working out more details yields a concrete region where $Z_{I}^{(N)}(s)$ is holomorphic.

Note that the locus of $D_{N}$ where it is not a normal crossings divisor, i.e., not locally monomial as in Lemma 3.1, consists of the points with at least two coordinates equal to 0 , at least two coordinates equal to 1 , or at least three equal coordinates. We will blow up consecutively in the relevant centres of increasing dimension contained in that locus, until the total inverse image of $D_{N}$ becomes a normal crossings divisor.

Proposition 5.1. We take $I=\{2, \ldots, N-2\}$. Then $Z_{I}^{(N)}(s)$ is convergent and holomorphic in a region containing $\operatorname{Re}\left(s_{i j}\right)>-\frac{2}{N-2}$ for all ij. In particular, the integral $Z_{I}^{(N)}(s)$ is holomorphic in the half-plane $\operatorname{Re}(s)>-\frac{2}{N-2}$.

Proof. We recall that

$$
Z_{I}^{(N)}(s):=\int_{\mathbb{R}^{N-3}} \varphi_{I}(x) F_{I}(x, s) \prod_{i=2}^{N-2} d x_{i},
$$


where

$$
F_{I}(x, s)=\prod_{j=2}^{N-2}\left|x_{j}\right|^{s_{1 j}} \prod_{j=2}^{N-2}\left|1-x_{j}\right|^{s_{(N-1) j}} \prod_{2 \leq i<j \leq N-2}\left|x_{i}-x_{j}\right|^{s_{i j}} .
$$

First, we consider an adequate partition of the unity subordinate to the compact set $\operatorname{supp} \varphi_{I}$. Let $P$ be the set of $2^{N-3}$ points $p$ in $\mathbb{R}^{N-3}$ with all coordinates equal to 0 or 1 . We take smooth functions $\Omega_{p}, p \in P$, such that each $\Omega_{p}$ is supported in a neighborhood of $p$ that is disjoint from some neighborhood of any other point of $P$, and such that $\varphi_{I}(x)=\sum_{p \in P} \Omega_{p}(x)$ for $x \in \operatorname{supp} \varphi_{I}$. Then

$$
Z_{I}^{(N)}(\boldsymbol{s})=\sum_{p \in P} Z_{\Omega_{p}}^{(N)}(\boldsymbol{s}), \quad \text { where } \quad Z_{\Omega_{p}}^{(N)}(\boldsymbol{s}):=\int_{\mathbb{R}^{N-3}} \Omega_{p}(x) F_{I}(x, s) \prod_{i=2}^{N-2} d x_{i} .
$$

(1) We start by improving the situation around the origin $(p=0)$. We remark that the factors $\left|1-x_{j}\right|^{s_{(N-1) j}}$ in the integrand of $Z_{\Omega_{0}}^{(N)}(s)$ are invertible on the support of $\Omega_{0}$, and can be neglected from the point of view of convergence and holomorphy of $Z_{\Omega_{0}}^{(N)}(s)$. Hence, we want in this stage an embedded resolution of the divisor $D_{0}$ given by the zero locus of

$$
\prod_{i=2}^{N-2} x_{i} \prod_{2 \leq i<j \leq N-2}\left(x_{i}-x_{j}\right)
$$

(1.1) The blow-up at the origin of $\mathbb{R}^{N-3}$ involves $N-3$ changes of variables of type $x_{i_{0}}=u_{i_{0}}, x_{i}=u_{i_{0}} u_{i}$ for $i \in\{2, \ldots, N-2\} \backslash\left\{i_{0}\right\}$ and some fixed $i_{0} \in\{2, \ldots, N-2\}$. This change of variables defines

$$
\sigma_{0}: \mathbb{R}^{N-3} \rightarrow \mathbb{R}^{N-3}: u \mapsto x .
$$

Without loss of generality, we may assume that $i_{0}=2$. Then $F_{I}(x, s) \circ \sigma_{0}$ equals

$$
\left|u_{2}\right|^{\sum_{j=2}^{N-2} s_{1 j}+\sum_{2 \leq i<j \leq N-2} s_{i j}} \prod_{j=3}^{N-2}\left|u_{j}\right|^{s_{1 j}} \prod_{j=3}^{N-2}\left|1-u_{j}\right|^{s_{(N-1) j}} \prod_{3 \leq i<j \leq N-2}\left|u_{i}-u_{j}\right|^{s_{i j}} g(u, s),
$$

where

$$
g(u, s):=\left|1-u_{2}\right|^{s_{(N-1) 2}} \prod_{j=3}^{N-2}\left|1-u_{2} u_{j}\right|^{s_{(N-1) j}}
$$

and

$$
\sigma_{0}^{*} \prod_{i=2}^{N-2} d x_{i}=\left|u_{2}\right|^{N-4} \prod_{i=2}^{N-2} d u_{i} .
$$

Hence, the contribution to $Z_{\Omega_{0}}^{(N)}(s)$ in the chart (5.11), with $i_{0}=2$, takes the form

$$
\begin{aligned}
& \int_{\mathbb{R}^{N-3}}\left(\Omega_{0} \circ \sigma_{0}\right)(u)\left|u_{2}\right|^{\sum_{j=2}^{N-2} s_{1 j}+\sum_{2 \leq i<j \leq N-2} s_{i j}+N-4} \prod_{j=3}^{N-2}\left|u_{j}\right|^{s_{1 j}} \prod_{j=3}^{N-2}\left|1-u_{j}\right|^{s_{(N-1) j}} \times \\
& \prod_{3 \leq i<j \leq N-2}\left|u_{i}-u_{j}\right|^{s_{i j}} g(u, s) \prod_{i=2}^{N-2} d u_{i},
\end{aligned}
$$


where the factor $g(u, s)$ is invertible on the support of $\Omega_{0} \circ \sigma_{0}$, and can be neglected from the point of view of convergence and holomorphy. The first new condition for convergence is thus

$$
\sum_{j=2}^{N-2} \operatorname{Re}\left(s_{1 j}\right)+\sum_{2 \leq i<j \leq N-2} \operatorname{Re}\left(s_{i j}\right)+N-3>0
$$

Simplifying all $s_{i j}$ to $s$, the contribution to $Z_{\Omega_{0}}^{(N)}(s)$ in this chart is

$$
\int_{\mathbb{R}^{N-3}}\left(\Omega_{0} \circ \sigma_{0}\right)(u)\left|u_{2}\right|^{\frac{(N-2)(N-3)}{2} s+N-4} \prod_{i=3}^{N-2}\left|u_{i}\right|^{s} \prod_{i=3}^{N-2}\left|1-u_{i}\right|^{s} \prod_{3 \leq i<j \leq N-2}\left|u_{i}-u_{j}\right|^{s} g(u, s) \prod_{i=2}^{N-2} d u_{i} .
$$

Up to negligible factors as $g(u, s)$, further blowing-ups/changes of variables, ultimately leading to monomial integrals as in Lemma 3.1, will not affect the variable $u_{2}$ anymore.

The smooth hypersurface, given by $u_{2}=0$, corresponds to a submanifold $E_{0}$ (as in Theorem 3.1) with numerical data

$$
\left(N_{f_{N}, 0}, v_{0}\right)=\left(\frac{(N-2)(N-3)}{2}, N-3\right), \quad \text { satisfying } \quad \frac{v_{0}}{N_{f_{N}, 0}}=\frac{2}{N-2} .
$$

Important to note is that $N_{f_{N}, 0}$ is equal to the multiplicity of $D_{N}$ at the origin, which, in the case of a hyperplane arrangement, is just the number of hyperplanes containing the origin. Also, $v_{0}$ is equal to the codimension of the origin in $\mathbb{R}^{N-3}$. This is a general fact: for any submanifold $E_{i}$ as in Theorem 3.1, created by a blow-up with centre $Y$, we have that $N_{f_{N}, i}$ is equal to the multiplicity of $D_{N}$ at (a generic point of) $Y$, being the number of hyperplanes containing $Y$, and that $v_{i}$ is equal to the codimension of $Y$ in the ambient space.

(1.2) The next blow-ups, in centres intersecting $E_{0}$, are at those centres of dimension 1 whose image by $\sigma_{0}$ contains the origin. There are two such centres visible in the present chart. The first one is $u_{3}=\ldots=u_{N-2}=0$ (this is the so-called strict transform of the line $x_{3}=\ldots=x_{N-2}=0$ ). This blow-up consists of $N-4$ changes of variables of type $u_{i_{1}}=w_{i_{1}}$, for some $i_{1} \in\{3, \ldots, N-2\}$ and $u_{i}=w_{i_{1}} w_{i}$ for $i \in\{3, \ldots, N-2\}, i \neq i_{1}$, and $u_{2}=w_{2}$. We pick $i_{1}=3$ (the other cases are treated in a similar way), and take thus $u_{2}=w_{2}, u_{3}=w_{3}$, and $u_{i}=w_{3} w_{i}$ for $i \in\{4, \ldots, N-2\}$, defining the change of variables

$$
\sigma_{1}: \mathbb{R}^{N-3} \rightarrow \mathbb{R}^{N-3}: w \mapsto u
$$

Then in this chart the contribution to $Z_{\Omega_{0}}^{(N)}(s)$ takes the form

$$
\begin{aligned}
& \int_{\mathbb{R}^{N-3}}\left(\Omega_{0} \circ \sigma_{0} \circ \sigma_{1}\right)(w)\left|w_{2}\right|^{\sum_{j=2}^{N-2} s_{1 j}+\sum_{2 \leq i<j \leq N-2} s_{i j}+N-4}\left|w_{3}\right|^{\sum_{j=3}^{N-2} s_{1 j}+\sum_{3 \leq i<j \leq N-2} s_{i j}+N-5} \times \\
& \prod_{j=4}^{N-2}\left|w_{j}\right|^{s_{1 j}} \prod_{4 \leq j \leq N-2}\left|1-w_{j}\right|^{s_{(N-1) j}} \prod_{4 \leq i<j \leq N-2}\left|w_{i}-w_{j}\right|^{s_{i j}} h(w, s) \prod_{i=2}^{N-2} d w_{i},
\end{aligned}
$$


where the factor $h(w, s)$ can be neglected from the point of view of convergence and holomorphy. The next condition for convergence is thus

$$
\sum_{j=3}^{N-2} \operatorname{Re}\left(s_{1 j}\right)+\sum_{3 \leq i<j \leq N-2} \operatorname{Re}\left(s_{i j}\right)+N-4>0 .
$$

Simplifying, the contribution to $Z_{\Omega_{0}}^{(N)}(s)$ is

$$
\begin{aligned}
& \int_{\mathbb{R}^{N-3}}\left(\Omega_{0} \circ \sigma_{0} \circ \sigma_{1}\right)(w)\left|w_{2}\right|^{\frac{(N-2)(N-3)}{2} s+N-4}\left|w_{3}\right|^{\frac{(N-3)(N-4)}{2} s+N-5} \times \\
& \prod_{i=4}^{N-2}\left|w_{i}\right|^{s} \prod_{4 \leq j \leq N-2}\left|1-w_{i}\right|^{s} \prod_{4 \leq i<j \leq N-2}\left|w_{i}-w_{j}\right|^{s} h(w, s) \prod_{i=2}^{N-2} d w_{i} .
\end{aligned}
$$

The smooth hypersurface, given by $w_{3}=0$, corresponds to a submanifold $E_{1}$ with numerical data

$$
\left(N_{f_{N}, 1}, v_{1}\right)=\left(\frac{(N-3)(N-4)}{2}, N-4\right), \quad \text { satisfying } \quad \frac{v_{1}}{N_{f_{N}, 1}}=\frac{2}{N-3} .
$$

The second centre is $1=u_{3}=\ldots=u_{N-2}$ (the strict transform of the line $x_{2}=x_{3}=$ $\left.\ldots=x_{N-2}\right)$. After a change of variables $u_{i}^{\prime}=u_{i}-1$ for $i=3, \ldots, N-2$, the calculation of this blow-up is the same as for the first centre. It gives rise to a submanifold $E_{1}^{\prime}$ with the same numerical data $\left(N_{f_{N}, 1}^{\prime}, v_{1}^{\prime}\right)=\left(\frac{(N-3)(N-4)}{2}, N-4\right)$, yielding the same quotient $\frac{v_{1}^{\prime}}{N_{f_{N}, 1}^{\prime}}=\frac{2}{N-3}$. The associated new condition for convergence for $Z_{\Omega_{0}}^{(N)}(s)$ is

$$
\sum_{2 \leq i<j \leq N-2} \operatorname{Re}\left(s_{i j}\right)+N-4>0
$$

(1.3) We continue this way, blowing up in centres of increasing dimension, ending with blow-ups in centres of dimension $N-5$ of two possible types, for instance corresponding to $x_{N-3}=x_{N-2}=0$ and $x_{N-4}=x_{N-3}=x_{N-2}$, respectively, yielding submanifolds with numerical data $(3,2)$. The corresponding conditions for convergence for $Z_{\Omega_{0}}^{(N)}(s)$ are

$$
\operatorname{Re}\left(s_{1(N-3)}\right)+\operatorname{Re}\left(s_{1(N-2)}\right)+\operatorname{Re}\left(s_{(N-3)(N-2)}\right)+2>0
$$

and

$$
\operatorname{Re}\left(s_{(N-4)(N-3)}\right)+\operatorname{Re}\left(s_{(N-4)(N-2)}\right)+\operatorname{Re}\left(s_{(N-3)(N-2)}\right)+2>0 .
$$

Note that, up to now, the smallest quotient of numerical data that we obtained is indeed $\frac{2}{N-2}$.

(2) All other points $p=\left(p_{2}, \ldots, p_{N-2}\right) \in P$, that are needed as centres of blow-ups, have at least one coordinate equal to 1 (and still at least two coordinates equal to 0 or at least two coordinates equal to 1 ), say $p_{i}=1$ for $i \in J \neq \emptyset$ and $p_{i}=0$ for $i \notin J$. For simplicity, 
we switch to the coordinate system $y$, given by $y_{i}=x_{i}-1$ for $i \in J$ and $y_{i}=x_{i}$ for $i \notin J$, in order to view $p$ as the new origin. Then

$$
\begin{aligned}
Z_{\Omega_{p}}^{(N)}(\boldsymbol{s})= & \int_{\mathbb{R}^{N-3}} \Omega_{p}(y) \prod_{i \notin J}\left|y_{i}\right|^{s_{1 j}} \prod_{i \in J}\left|y_{i}\right|^{s_{(N-1) j}} \times \\
& \prod_{\substack{2 \leq i<j \leq N-2 \\
i, j \in J}}\left|y_{i}-y_{j}\right|^{s_{i j}} \prod_{\substack{2 \leq i<j \leq N-2 \\
i, j \notin J}}\left|y_{i}-y_{j}\right|^{s_{i j}} g_{p}(y, s) \prod_{i=2}^{N-2} d y_{i},
\end{aligned}
$$

where $g_{p}(y, s)$ is an invertible function on the support of $\Omega_{p}(y)$, smooth in $y$ and holomorphic in $s$. This simplifies to

$$
Z_{\Omega_{p}}^{(N)}(s)=\int_{\mathbb{R}^{N-3}} \Omega_{p}(y) \prod_{i \in I}\left|y_{i}\right|^{s} \prod_{\substack{2 \leq i<j \leq N-2 \\ i, j \in J}}\left|y_{i}-y_{j}\right|^{s} \prod_{\substack{2 \leq i<j \leq N-2 \\ i, j \notin J}}\left|y_{i}-y_{j}\right|^{s} g_{p}(y, s) \prod_{i=2}^{N-2} d y_{i}
$$

The divisor $D_{p}$ attached to (5.18) is given by the zero locus of

$$
\prod_{i \in I} y_{i} \prod_{\substack{2 \leq i<j \leq N-2 \\ i, j \in J}}\left(y_{i}-y_{j}\right) \prod_{\substack{2 \leq i<j \leq N-2 \\ i, j \notin J}}\left(y_{i}-y_{j}\right) .
$$

It can be considered as a subarrangement of the arrangement $D_{0}$. Hence, an embedded resolution of $D_{p}$ can be constructed by (part of) the same blow-ups we used to construct the embedded resolution of $D_{0}$. Take any centre of blow-up $Z_{i}$, of codimension $v_{i}$, occurring in those resolutions, leading to the exceptional submanifold $E_{i}$. Say $n_{i}$ and $n_{i}^{\prime}$ are the number of hyperplanes in $D_{0}$ and $D_{p}$, respectively, containing $Z_{i}$; then clearly $n_{i}^{\prime} \leq n_{i}$. Hence the numerical data of $E_{i}$, considered in the embedded resolution of $D_{0}$ and $D_{p}$, are $\left(n_{i}, v_{i}\right)$ and $\left(n_{i}^{\prime}, v_{i}\right)$, respectively. Since $\frac{v_{i}}{n_{i}} \leq \frac{v_{i}}{n_{i}^{\prime}}$, all new quotients of numerical data are again at least $\frac{2}{N-2}$. For the original integral $Z_{\Omega_{p}}^{(N)}(s)$, we get new conditions for convergence similar to (5.12), (5.14), (5.15) till (5.17), involving the same constant terms, but with other variables $s_{i j}$, still all having coefficient 1 , and with at most the number of variables $s_{i j}$ as in those expressions.

In particular, if $\operatorname{Re}\left(s_{i j}\right)>-\frac{2}{N-2}$ for all $i j$, then all convergence conditions are satisfied. (The 'hardest' type is (5.12), when $\frac{(N-2)(N-3)}{2}$ variables $s_{i j}$ occur.)

\subsection{Case $I \neq\{2, \ldots, N-2\}$}

In this case, recall that $Z_{I}^{(N)}(s)$ takes the form

$$
Z_{I}^{(N)}(s)=\int_{\mathbb{R}^{N-3}} \widetilde{\varphi}_{I}(x) \prod_{i \notin I}\left|x_{i}\right|^{-s_{1 i}-s_{(N-1) i}-\sum_{2 \leq j \leq N-2} s_{\substack{j \neq \\ j \neq i}}^{s_{i j}-2}} F_{I}(x, s) \prod_{i=2}^{N-2} d x_{i}
$$


where

$$
\begin{aligned}
F_{I}(x, s):= & \prod_{j \in I}\left|x_{j}\right|^{s_{1 j}} \prod_{j=2}^{N-2}\left|1-x_{j}\right|^{s_{(N-1) j}} \prod_{\substack{2 \leq i<j \leq N-2 \\
i, j \in I}}\left|x_{i}-x_{j}\right|^{s_{i j}} \times \\
& \prod_{\substack{i \leq i<j \leq N-2 \\
i, j \notin I}}\left|x_{i}-x_{j}\right|^{s_{i j}} \prod_{\substack{2 \leq i<j \leq N-2 \\
i \notin I, j \in I}}\left|1-x_{i} x_{j}\right|^{s_{i j}} \prod_{\substack{2 \leq i<j \leq N-2 \\
i \in I, j \notin I}}\left|1-x_{i} x_{j}\right|^{s_{i j}} .
\end{aligned}
$$

This simplifies to

$$
Z_{I}^{(N)}(s):=\int_{\mathbb{R}^{N-3}} \widetilde{\varphi}_{I}(x) \prod_{i \notin I}\left|x_{i}\right|^{-(N-2) s-2} F_{I}(x, s) \prod_{i=2}^{N-2} d x_{i} .
$$

The relevant arrangement here is the divisor $D_{I, 0} \cup D_{I}$, where $D_{I, 0}:=\left\{\prod_{i \notin I} x_{i}=0\right\}$ and $D_{I}$ is the zero locus of

$$
\begin{gathered}
\prod_{i \in I} x_{i} \prod_{i=2}^{N-2}\left(1-x_{i}\right) \prod_{\substack{2 \leq i<j \leq N-2 \\
i, j \notin I}}\left(x_{i}-x_{j}\right) \prod_{\substack{2 \leq i<j \leq N-2 \\
i, j \in I}}\left(x_{i}-x_{j}\right) \times \\
\prod_{\substack{2 \leq i<j \leq N-2 \\
i \notin I, j \in I}}\left(1-x_{i} x_{j}\right) \prod_{\substack{2 \leq i<j \leq N-2 \\
i \in I, j \notin I}}\left(1-x_{i} x_{j}\right) .
\end{gathered}
$$

Proposition 5.2. Fix $I \neq\{2, \ldots, N-2\}$. Then $Z_{I}^{(N)}(s)$ is convergent and holomorphic in a region containing $-\frac{2}{N-2}<\operatorname{Re}\left(s_{i j}\right)<-\frac{2}{N}$ for all ij. In particular, the integral $Z_{I}^{(N)}(s)$ is holomorphic in the band $-\frac{2}{N-2}<\operatorname{Re}(s)<-\frac{2}{N}$.

Proof. Note first that in this case the divisors $D_{I}$ and $D_{I, 0}$ play a different role. All components of (the strict transforms of) $D_{I}$ induce the classical condition $\operatorname{Re}\left(s_{i j}\right)+1>$ $0 \Leftrightarrow \operatorname{Re}\left(s_{i j}\right)>-1$. But the components of $D_{I, 0}$ give rise to a new kind of convergence condition of type

$$
-\operatorname{Re}\left(s_{1 i}\right)-\operatorname{Re}\left(s_{(N-1) i}\right)-\sum_{\substack{2 \leq j \leq N-2 \\ j \neq i}} \operatorname{Re}\left(s_{i j}\right)-1>0,
$$

simplifying for $Z_{I}^{(N)}(s)$ to

$$
-(N-2) \operatorname{Re}(s)-1>0 \Leftrightarrow \operatorname{Re}(s)<-\frac{1}{N-2} .
$$

Next, we construct an embedded resolution of $D_{I} \cup D_{I, 0}$. A crucial observation is that any blow-up with centre not contained in $D_{I, 0}$ will induce a convergence condition that already appeared in the construction of the resolution $\sigma$ in the proof of Proposition 5.1. In particular, when $-\frac{2}{N-2}<\operatorname{Re}\left(s_{i j}\right)$ for all $i j$, then all such conditions are satisfied. For $Z_{I}^{(N)}(s)$, these conditions get the form $-\frac{2}{N-2}<\operatorname{Re}(s)$ or weaker. We could make this lower 
bound more precise, depending on the size of $I$, but this would not affect the end result of Theorem 4.1.

We now treat the blow-ups with centre in $D_{I, 0}$. In particular, we will show that they induce for $Z_{I}^{(N)}(s)$ as strongest condition $\operatorname{Re}(s)<-\frac{2}{N}$.

In a small enough neighborhood of $D_{I, 0}$, we can write the integrand in $Z_{I}^{(N)}(s)$ in the form

$$
\begin{aligned}
& \widetilde{\varphi}_{I}(x) \prod_{i \notin I}\left|x_{i}\right|^{-s_{1 i}-s_{(N-1) i}-\sum_{2 \leq j \leq N-2} s_{i j}-2} \prod_{\substack{j \neq i \\
2 \leq i<j \leq N-2 \\
i, j \notin I}}\left|x_{i}-x_{j}\right|^{s_{i j}} \\
& \prod_{j \in I}\left|x_{j}\right|^{s_{1 j}} \prod_{j=2}^{N-2}\left|1-x_{j}\right|^{s_{(N-1) j}} \prod_{\substack{2 \leq i<j \leq N-2 \\
i, j \in I}}\left|x_{i}-x_{j}\right|^{s_{i j}} g(x, s),
\end{aligned}
$$

where the factor $g(x, s)$ is invertible on the support of $\widetilde{\varphi}_{I}(x)$ (and can be neglected from the point of view of convergence and holomorphy). This simplifies for the integrand in $Z_{I}^{(N)}(s)$ to

$$
\begin{aligned}
& \widetilde{\varphi}_{I}(x) \prod_{i \notin I}\left|x_{i}\right|^{-(N-2) s-2} \prod_{\substack{2 \leq i<j \leq N-2 \\
i, j \notin I \\
i, j \notin I}}\left|x_{i}-x_{j}\right|^{s} \times \\
& \prod_{i \in I}\left|x_{i}\right|^{s} \prod_{i \in I}\left|1-x_{i}\right|^{s} \prod_{\substack{2 \leq i<j \leq N-2 \\
i, j \in I}}\left|x_{i}-x_{j}\right|^{s} g(x, s) .
\end{aligned}
$$

After a permutation of the indices, we may assume that $I=\{2, \ldots, N-2\} \backslash\{2, \ldots, l\}$ with $l \geq 2$. Then $D_{I, 0}$ is given by $\prod_{2 \leq i \leq l} x_{i}=0$. When $l=2$ (i.e., $|I|=N-4$ ), no blow-up with centre in $D_{I, 0}$ is needed. If $l \geq 3$ (i.e., $|I| \leq N-5$ ), we start by performing a blow-up $\tau$ with centre at $x_{2}=\ldots=x_{l}=0$, for instance in the chart $x_{2}=u_{2}, x_{i}=u_{i} u_{2}$ for $i=3, \ldots, l$, and $x_{i}=u_{i}$ for $i=l+1, \ldots, N-2$. This centre is contained in the $l-1$ hyperplanes $x_{i}=0,2 \leq i \leq l$, and in the corresponding $\frac{(l-1)(l-2)}{2}$ hyperplanes $x_{i}-x_{j}=0$, with $2 \leq i, j \leq l$. Hence the power of $\left|u_{2}\right|$ in the pullback of the integrand in $Z_{I}^{(N)}(s)$ is

$$
\sum_{i=2}^{l}\left(-s_{1 i}-s_{(N-1) i}-\sum_{\substack{2 \leq j \leq N-2 \\ j \neq i}} s_{i j}-2\right)+\sum_{2 \leq i<j \leq l} s_{i j}+(l-2),
$$

which we rewrite as

$$
-\sum_{i=2}^{l}\left(s_{1 i}+s_{(N-1) i}\right)-\sum_{i=2}^{l} \sum_{j=l+1}^{N-2} s_{i j}-\sum_{2 \leq i<j \leq l} s_{i j}-l .
$$

Note the important fact that all coefficients of the variables are -1 . The clue in the rewriting above is that, for $s_{i j}$ with $2 \leq i<j \leq l$, we had twice the coefficient +1 (coming from $x_{i}$ and $x_{j}$ ), and once the coefficient -1 (coming from $x_{i}-x_{j}$ ). The associated new condition for convergence for $Z_{I}^{(N)}(s)$ is thus

$$
-\sum_{i=2}^{l}\left(\operatorname{Re}\left(s_{1 i}\right)+\operatorname{Re}\left(s_{(N-1) i}\right)\right)-\sum_{i=2}^{l} \sum_{j=l+1}^{N-2} \operatorname{Re}\left(s_{i j}\right)-\sum_{2 \leq i<j \leq l} \operatorname{Re}\left(s_{i j}\right)-l+1>0 .
$$


Note that $2(l-1)+(l-1)(N-l-2)+\frac{(l-1)(l-2)}{2}=(l-1) \frac{(2 N-l-2)}{2}$ different variables occur. Hence, for $Z_{I}^{(N)}(s)$, this simplifies to $\left.l-1\right) \frac{(2 N-l-2)}{2} \operatorname{Re}(s)-(l-1)>0$, i.e.,

$$
\operatorname{Re}(s)<-\frac{2}{2 N-l-2} .
$$

This condition is the strongest when $l=N-2$ (i.e., $I=\emptyset$ ), and becomes then $\operatorname{Re}(s)<-\frac{2}{N}$. In that case $(5.22)$ is

$$
-\sum_{i=2}^{N-2}\left(\operatorname{Re}\left(s_{1 i}\right)+\operatorname{Re}\left(s_{(N-1) i}\right)\right)-\sum_{2 \leq i<j \leq N-2} \operatorname{Re}\left(s_{i j}\right)-N+3>0 .
$$

Further blow-ups to make the integrand locally monomial, for instance with centre $u_{3}=\cdots=u_{l}=0$ in the chart above, yield completely similar conditions. In fact, after another permutation of the indices, we obtain again the condition (5.22), but for smaller $l$.

In particular, if $\operatorname{Re}\left(s_{i j}\right)<-\frac{2}{N}$ for all $i j$, then all convergence conditions (5.19) and type (5.22) are satisfied. (The 'hardest' type is (5.24), due to the largest number of occurring variables.) For $Z_{I}^{(N)}(s)$, it is indeed clear that $\operatorname{Re}(s)<-\frac{2}{N}$ is a stronger condition than (5.20).

\subsection{Proof of Theorem 4.1 and precise description of the convergence domain}

The statement of Theorem 4.1 now follows quite immediately.

Proof. From Propositions 5.1 and 5.2, we know that the Koba-Nielsen local zeta function $Z_{\mathbb{R}}^{(N)}(s)$ is convergent and holomorphic in a region containing $-\frac{2}{N-2}<\operatorname{Re}\left(s_{i j}\right)<-\frac{2}{N}$ for all $i j$. This is enough to imply meromorphic continuation to the whole $\mathbb{C}^{N-3}$.

The restrictions concerning the numbers $N_{i j, k}(I)$ and $\gamma_{k}(I)$ in the actual convergence domain $\cap_{I} \mathcal{H}(I)$, see (4.9), are a consequence of the proofs of these propositions.

Moreover, the details in the strategy and outline of the proofs of Propositions 5.1 and 5.2 yield the following more precise description of the actual convergence domain. Below, the inequalities (5.26) generalize $(5.12),(5.14), \ldots,(5.16)$, where the set $J$ is $\{2, \ldots, N-2\},\{3, \ldots, N-2\}, \ldots,\{N-3, N-2\}$, respectively. The inequalities (5.27) are the similar ones according to the symmetry $1 \leftrightarrow N-1$ in the integrand of $Z_{\mathbb{R}}^{(N)}(s)$, corresponding geometrically to $(0,0, \ldots, 0) \leftrightarrow(1,1, \ldots, 1)$. Next, the inequalities (5.28) generalize $(5.15), \ldots,(5.17)$, where $J$ is $\{2, \ldots, N-2\},\{3, \ldots, N-2\}, \ldots,\{N-4, N-$ $3, N-2\}$, respectively. Finally, in (5.29) the sets $J$ generalize $\{i\}$ in $(5.19)$ and $\{2, \ldots, l\}$ in (5.22).

Proposition 5.3. The Koba-Nielsen local zeta function $Z_{\mathbb{R}}^{(N)}(s)$ is convergent and holomorphic in the region determined by the following $2^{N-1}-N-1$ inequalities:

$$
\operatorname{Re}\left(s_{i j}\right)>-1
$$

for all $\frac{N(N-3)}{2}$ variables $s_{i j}$,

$$
\sum_{j \in J} \operatorname{Re}\left(s_{1 j}\right)+\sum_{\substack{i, j \in J \\ i<j}} \operatorname{Re}\left(s_{i j}\right)>-\sharp J
$$


for all subsets $J \subset\{2, \ldots, N-2\}$ with $\sharp J \geq 2$,

$$
\sum_{j \in J} \operatorname{Re}\left(s_{(N-1) j}\right)+\sum_{\substack{i, j \in J \\ i<j}} \operatorname{Re}\left(s_{i j}\right)>-\sharp J
$$

for all subsets $J \subset\{2, \ldots, N-2\}$ with $\sharp J \geq 2$,

$$
\sum_{\substack{i, j \in J \\ i<j}} \operatorname{Re}\left(s_{i j}\right)>-\sharp J+1
$$

for all subsets $J \subset\{2, \ldots, N-2\}$ with $\sharp J \geq 3$,

$$
\sum_{j \in J} \operatorname{Re}\left(s_{1 j}\right)+\sum_{j \in J} \operatorname{Re}\left(s_{(N-1) j}\right)+\sum_{\substack{i \in\{2, \ldots, N-2\} \backslash J \\ j \in J}} \operatorname{Re}\left(s_{i j}\right)+\sum_{\substack{i, j \in J \\ i<j}} \operatorname{Re}\left(s_{i j}\right)<-\sharp J
$$

for all subsets $J \subset\{2, \ldots, N-2\}$ with $\sharp J \geq 1$.

\subsection{Example}

Fix $N=6$. Following the proofs of Propositions 5.1 and 5.2, or as special case of Proposition 5.3 , we obtain that $Z^{(6)}(s)$ is holomorphic in the region

$$
\begin{aligned}
(\text { basic }) & \operatorname{Re}\left(s_{i j}\right)>-1 \quad \text { for all nine } i j, \\
(I=\{2,3.4\}) & \operatorname{Re}\left(s_{12}\right)+\operatorname{Re}\left(s_{13}\right)+\operatorname{Re}\left(s_{14}\right)+\operatorname{Re}\left(s_{23}\right)+\operatorname{Re}\left(s_{24}\right)+\operatorname{Re}\left(s_{34}\right)>-3, \\
& \operatorname{Re}\left(s_{52}\right)+\operatorname{Re}\left(s_{53}\right)+\operatorname{Re}\left(s_{54}\right)+\operatorname{Re}\left(s_{23}\right)+\operatorname{Re}\left(s_{24}\right)+\operatorname{Re}\left(s_{34}\right)>-3, \\
& \operatorname{Re}\left(s_{12}\right)+\operatorname{Re}\left(s_{13}\right)+\operatorname{Re}\left(s_{23}\right)>-2, \quad \operatorname{Re}\left(s_{12}\right)+\operatorname{Re}\left(s_{14}\right)+\operatorname{Re}\left(s_{24}\right)>-2, \\
& \operatorname{Re}\left(s_{13}\right)+\operatorname{Re}\left(s_{14}\right)+\operatorname{Re}\left(s_{34}\right)>-2, \quad \operatorname{Re}\left(s_{52}\right)+\operatorname{Re}\left(s_{53}\right)+\operatorname{Re}\left(s_{23}\right)>-2, \\
& \operatorname{Re}\left(s_{52}\right)+\operatorname{Re}\left(s_{54}\right)+\operatorname{Re}\left(s_{24}\right)>-2, \quad \operatorname{Re}\left(s_{53}\right)+\operatorname{Re}\left(s_{54}\right)+\operatorname{Re}\left(s_{34}\right)>-2, \\
& \operatorname{Re}\left(s_{23}\right)+\operatorname{Re}\left(s_{24}\right)+\operatorname{Re}\left(s_{34}\right)>-2, \\
(|I|<3) & \operatorname{Re}\left(s_{12}\right)+\operatorname{Re}\left(s_{52}\right)+\operatorname{Re}\left(s_{23}\right)+\operatorname{Re}\left(s_{24}\right)<-1, \\
& \operatorname{Re}\left(s_{13}\right)+\operatorname{Re}\left(s_{53}\right)+\operatorname{Re}\left(s_{23}\right)+\operatorname{Re}\left(s_{34}\right)<-1, \\
& \operatorname{Re}\left(s_{14}\right)+\operatorname{Re}\left(s_{54}\right)+\operatorname{Re}\left(s_{24}\right)+\operatorname{Re}\left(s_{34}\right)<-1, \\
& \operatorname{Re}\left(s_{12}\right)+\operatorname{Re}\left(s_{52}\right)+\operatorname{Re}\left(s_{13}\right)+\operatorname{Re}\left(s_{53}\right)+\operatorname{Re}\left(s_{23}\right)+\operatorname{Re}\left(s_{24}\right)+\operatorname{Re}\left(s_{34}\right)<-2, \\
& \operatorname{Re}\left(s_{12}\right)+\operatorname{Re}\left(s_{52}\right)+\operatorname{Re}\left(s_{14}\right)+\operatorname{Re}\left(s_{54}\right)+\operatorname{Re}\left(s_{23}\right)+\operatorname{Re}\left(s_{24}\right)+\operatorname{Re}\left(s_{34}\right)<-2, \\
& \operatorname{Re}\left(s_{13}\right)+\operatorname{Re}\left(s_{53}\right)+\operatorname{Re}\left(s_{14}\right)+\operatorname{Re}\left(s_{54}\right)+\operatorname{Re}\left(s_{23}\right)+\operatorname{Re}\left(s_{24}\right)+\operatorname{Re}\left(s_{34}\right)<-2, \\
& \sum_{i j} \operatorname{Re}\left(s_{i j}\right)<-3,
\end{aligned}
$$

containing the simpler domain $-\frac{1}{2}<\operatorname{Re}\left(s_{i j}\right)<-\frac{1}{3}$ for all $i j$. 


\section{Local zeta functions of Koba-Nielsen type over local fields}

The Koba-Nielsen local zeta functions introduced in Definition 1 can be naturally defined over arbitrary local fields of characteristic zero, i.e., $\mathbb{R}, \mathbb{C}$, or finite extensions of $\mathbb{Q}_{p}$, the field of $p$-adic numbers, and the proof of the main theorem can be extended easily to the case of local fields different from $\mathbb{R}$. We denote the corresponding local zeta functions as $Z_{\mathbb{K}}^{(N)}(s)$ to emphasize the dependency on $\mathbb{K}$. Note that the $p$-adic case was already treated in [11]-[12] through an alternative method, only available in that case, called Igusa's stationary phase formula.

\subsection{Local fields}

We take $\mathbb{K}$ to be a non-discrete locally compact field of characteristic zero. Then $\mathbb{K}$ is $\mathbb{R}$, $\mathbb{C}$, or a finite extension of $\mathbb{Q}_{p}$, the field of $p$-adic numbers. If $\mathbb{K}$ is $\mathbb{R}$ or $\mathbb{C}$, we say that $\mathbb{K}$ is an $\mathbb{R}$-field, otherwise we say that $\mathbb{K}$ is a $p$-field.

For $a \in \mathbb{K}$, we define the modulus $|a|_{\mathbb{K}}$ of $a$ by

$$
|a|_{\mathbb{K}}=\left\{\begin{array}{l}
\text { the rate of change of the Haar measure in }(\mathbb{K},+) \text { under } x \rightarrow a x \\
\text { for } a \neq 0 \\
0 \text { for } a=0
\end{array}\right.
$$

It is well-known that, if $\mathbb{K}$ is an $\mathbb{R}$-field, then $|a|_{\mathbb{R}}=|a|$ and $|a|_{\mathbb{C}}=|a|^{2}$, where $|\cdot|$ denotes the usual absolute value in $\mathbb{R}$ or $\mathbb{C}$, and, if $\mathbb{K}$ is a $p$-field, then $|\cdot|_{\mathbb{K}}$ is the normalized absolute value in $\mathbb{K}$.

We now take $\mathbb{K}$ to be a $p$-field. Let $R_{\mathbb{K}}$ be the valuation ring of $\mathbb{K}, P_{\mathbb{K}}$ the maximal ideal of $R_{\mathbb{K}}$, and $\overline{\mathbb{K}}=R_{\mathbb{K}} / P_{\mathbb{K}}$ the residue field of $\mathbb{K}$. The cardinality of the residue field of $\mathbb{K}$ is denoted by $q$, thus $\overline{\mathbb{K}}=\mathbb{F}_{q}$. For $z \in \mathbb{K}$, ord $(z) \in \mathbb{Z} \cup\{+\infty\}$ denotes the valuation of $z$, and $|z|_{\mathbb{K}}=q^{-\operatorname{ord}(z)}$. We fix a uniformizing parameter $\mathfrak{p}$ of $R_{\mathbb{K}}$, i.e., a generator of $P_{\mathbb{K}}$.

We fix a set $S_{\mathbb{K}} \subset R_{\mathbb{K}}$ of representatives of the residue field $\overline{\mathbb{K}}$. We assume that $0 \in S_{\mathbb{K}}$. Any $z \in \mathbb{K} \backslash\{0\}$ admits a power expansion of the form

$$
z=\mathfrak{p}^{m} \sum_{k=0}^{\infty} z_{k} \mathfrak{p}^{k},
$$

where $m \in \mathbb{Z}$, the $z_{k}$ belong to $S_{\mathbb{K}}$, and $z_{0} \neq 0$. The series $(6.1)$ converges in the norm $|\cdot|_{\mathbb{K}}$.

\subsection{Multivariate local zeta functions: general case}

If $\mathbb{K}$ is a $p$-field, resp. an $\mathbb{R}$-field, we denote by $\mathcal{D}\left(\mathbb{K}^{n}\right)$ the $\mathbb{C}$-vector space consisting of all $\mathbb{C}$-valued locally constant functions, resp. all smooth functions, on $\mathbb{K}^{n}$, with compact support. An element of $\mathcal{D}\left(\mathbb{K}^{n}\right)$ is called a test function.

Let $f_{1}(x), \ldots, f_{m}(x) \in \mathbb{K}\left[x_{1}, \ldots, x_{n}\right]$ be non-constant polynomials, we denote by $D_{\mathbb{K}}:=$ $\cup_{i=1}^{m} f_{i}^{-1}(0)$ the divisor attached to them. We set

$$
\boldsymbol{f}:=\left(f_{1}, \ldots, f_{m}\right) \text { and } s:=\left(s_{1}, \ldots, s_{m}\right) \in \mathbb{C}^{m}
$$


The multivariate local zeta function attached to $(\boldsymbol{f}, \Theta)$, with $\Theta \in \mathcal{D}\left(\mathbb{K}^{n}\right)$, is defined as

$$
Z_{\Theta}(\boldsymbol{f}, \boldsymbol{s})=\int_{\mathbb{K}^{n} \backslash D_{\mathbb{K}}} \Theta(x) \prod_{i=1}^{m}\left|f_{i}(x)\right|_{\mathbb{K}}^{s_{i}} \prod_{i=1}^{n} d x_{i}, \quad \text { when } \operatorname{Re}\left(s_{i}\right)>0 \text { for all } i \text {. }
$$

Integrals of type (6.2) are analytic functions, and they admit meromorphic continuations to the whole $\mathbb{C}^{m}$, see $[37-39,46]$. By applying Hironaka's resolution of singularities theorem to $D_{\mathbb{K}}$, the study of integrals of type (6.2) is reduced to the case of monomial integrals, which can be studied directly, see e.g. [37, 38, 46].

Lemma 6.1. Let $\Phi\left(y, s_{1}, \ldots, s_{m}\right)$ be a test function with support in the polydisc

$$
\left\{y \in \mathbb{K}^{n} ;\left|y_{i}\right|<1, \text { for } i=1, \ldots, n\right\},
$$

when $\mathbb{K}$ is an $\mathbb{R}$-field, and with support $\mathfrak{p}^{e} R_{\mathbb{K}}^{n}(e \in \mathbb{Z})$ when $\mathbb{K}$ is a p-field, which is holomorphic in $s_{1}, \ldots, s_{m}$. Consider the integral

$$
J_{\mathbb{K}}\left(s_{1}, \ldots, s_{m}\right)=\int_{\mathbb{K}^{n}} \Phi\left(y, s_{1}, \ldots, s_{m}\right) \prod_{i=1}^{r}\left|y_{i}\right|_{\mathbb{K}}^{\sum_{j=1}^{m} a_{j, i} s_{j}+b_{i}-1} \prod_{i=1}^{n} d y_{i},
$$

where $1 \leq r \leq n$, for each $i$ the $a_{j, i}$ are integers (not all zero) and $b_{i}$ is an integer. Set

$$
\mathcal{R}_{\mathbb{K}}:=\bigcap_{i \in\{1, \ldots, r\}}\left\{\left(s_{1}, \ldots, s_{m}\right) \in \mathbb{C}^{m} ; \sum_{j=1}^{m} a_{j, i} \operatorname{Re}\left(s_{j}\right)+b_{i}>0\right\} .
$$

Then the following assertions hold:

(i) if all the $a_{j, i}$ are nonnegative integers (not all zero) and $b_{i}$ is a positive integer, then $\mathcal{R}_{\mathbb{K}} \neq \emptyset$. More precisely, $\left\{\left(s_{1}, \ldots, s_{m}\right) \in \mathbb{C}^{m} ; \operatorname{Re}\left(s_{j}\right)>0, j=1, \cdots, m\right\} \subset \mathcal{R}_{\mathbb{K}} ;$

(ii) if $\mathcal{R}_{\mathbb{K}} \neq \emptyset$, then $J_{\mathbb{K}}\left(s_{1}, \ldots, s_{m}\right)$ is convergent and defines a holomorphic function in the domain $\mathcal{R}_{\mathbb{K}}$. Moreover, in the $p$-field case, it is a rational function in $q^{-s_{1}}, \ldots, q^{-s_{m}}$;

(ii) if $\mathcal{R}_{\mathbb{K}} \neq \emptyset$, then the function $J_{\mathbb{K}}\left(s_{1}, \ldots, s_{m}\right)$ admits an analytic continuation to the whole $\mathbb{C}^{m}$, as a meromorphic function with poles belonging to

$$
\bigcup_{1 \leq i \leq r} \bigcup_{t}\left\{\sum_{j=1}^{m} a_{j, i} s_{j}+b_{i}+t=0\right\}
$$

in the $\mathbb{R}$-field case, with $t \in \mathbb{N}$ if $\mathbb{K}=\mathbb{R}$ and $t \in \frac{1}{2} \mathbb{N}$ if $\mathbb{K}=\mathbb{C}$, and with poles belonging to

$$
\bigcup_{1 \leq i \leq r}\left\{\sum_{j=1}^{m} a_{j, i} \operatorname{Re}\left(s_{j}\right)+b_{i}=0\right\}
$$

in the p-field case.

Also, Remark 1 extends to this more general setting. 
Remark 2. Theorem 3.2 then extends in an obvious way to this more general setting. In addition, in the $p$-field case, the integral $Z_{\Theta}(\boldsymbol{f}, \boldsymbol{s})$ admits a meromorphic continuation as a rational function

$$
Z_{\Theta}(\boldsymbol{f}, \boldsymbol{s})=\frac{P_{\Theta}(s)}{\left.\prod_{i \in T}\left(1-q^{-\left(\sum_{j=1}^{m} N_{f_{j}, i} s_{j}+v_{i}\right.}\right)\right)}
$$

in $q^{-s_{1}}, \ldots, q^{-s_{m}}$, where $P_{\Theta}(s)$ is a polynomial in the variables $q^{-s_{i}}$, and the real parts of its poles belong to the finite union of hyperplanes

$$
\sum_{j=1}^{m} N_{f_{j}, i} s_{j}+v_{i}=0, \quad \text { for } i \in T
$$

cf. ([46], Théorème 1.1.4.).

\subsection{Meromorphic continuation of local zeta functions: general case}

Theorem 6.1. Let $\mathbb{K}$ be a local field of characteristic zero. The Koba-Nielsen local zeta function $Z_{\mathbb{K}}^{(N)}(s)$ is a holomorphic function in the solution set $\cap_{I} \mathcal{H}(I)$, see $(4.9)$, in $\mathbb{C}^{\mathbf{d}}$, which contains the set

$$
-\frac{2}{N-2}<\operatorname{Re}\left(s_{i j}\right)<-\frac{2}{N} \quad \text { for all } i j .
$$

Furthermore, it has a meromorphic continuation, denoted again as $Z_{\mathbb{K}}^{(N)}(s)$, to the whole $\mathbb{C}^{\mathbf{d}}$. If $\mathbb{K}$ is an $\mathbb{R}$-field, the poles belong to $\cup_{I} \mathcal{P}(I)$, see (4.10), where now $t \in \frac{1}{2} \mathbb{N}$ for $\mathbb{K}=\mathbb{C}$. If $\mathbb{K}$ is a p-field, then this meromorphic continuation is a rational function in the variables $q^{-s_{i j}}$, with poles having real parts belonging to

$$
\bigcup_{k \in T(I)}\left\{s_{i j} \in \mathbb{R}^{\mathbf{d}} ; \sum_{i j \in M(I)} N_{i j, k}(I) s_{i j}+\gamma_{k}(I)=0\right\},
$$

where $N_{i j, k}(I), \gamma_{k}(I) \in \mathbb{Z}$, and $M(I), T(I)$ are finite sets. More precisely, for each $k$, either all numbers $N_{i j, k}(I)$ are equal to 0 or 1 and $\gamma_{k}(I)>0$, or all numbers $N_{i j, k}(I)$ are equal to 0 or -1 and $\gamma_{k}(I)<0$.

The proof of Theorem 6.1 is a slight variation of the proof of Theorem 4.1. We just indicate the required modifications. The first step is to express $Z_{\mathbb{K}}^{(N)}(s)$ as a finite sum of multivariate local zeta functions, see (4.6). This requires introducing an analogue of the functions $\chi$, see (4.4), and $\varphi_{I}$, see (4.5). We first define the analogue of $\chi$ in the complex case. We recall that an element of $\mathcal{D}\left(\mathbb{C}^{n}\right)$ is a $C^{\infty}$ function in the variables $z_{1}, \overline{z_{1}}, \ldots, z_{n}, \overline{z_{n}}$ (or in $\operatorname{Re}\left(z_{1}\right), \operatorname{Im}\left(z_{1}\right), \ldots, \operatorname{Re}\left(z_{n}\right), \operatorname{Im}\left(z_{n}\right)$ ). We pick, for $z=x+i y(x, y \in \mathbb{R}$ ),

$$
\chi_{\mathbb{C}}(z):=\chi\left(|z|_{\mathbb{C}}\right)=\chi\left(x^{2}+y^{2}\right),
$$


where $\chi$ is defined as in (4.4). Then $\chi_{\mathbb{C}}$ is a $C^{\infty}$ function in the variables $x, y$ satisfying

$$
\chi_{\mathbb{C}}(z)=\left\{\begin{array}{l}
1 \text { if } 0 \leq|z|_{\mathbb{C}} \leq 2 \\
0 \text { if }|z|_{\mathbb{C}} \geq 2+\epsilon .
\end{array}\right.
$$

We now define the function $\varphi_{I}$ as in (4.5). In the $p$-field case, we use

$$
\chi_{\mathfrak{p}}(z)=\left\{\begin{array}{l}
1 \text { if }|z|_{\mathbb{K}} \leq 1 \\
0 \text { if }|z|_{\mathbb{K}}>1
\end{array}\right.
$$

Now the proof follows line by line the one given for Theorem 4.1. This is possible because, for any $\mathbb{K}$, all the required blow-ups and centres are defined over the field of rational numbers.

Remark 3. Note that the convergence domains of $Z_{\mathbb{R}}^{(N)}(s)$ and $Z_{\mathbb{C}}^{(N)}(s)$ are exactly the same, due to the conventions $|a|_{\mathbb{R}}=|a|(a \in R)$ and $|a|_{\mathbb{C}}=|a|^{2}(a \in C)$ in the defining integrals.

The actual convergence domain of $Z_{\mathbb{C}}^{(N)}(s)$ can thus be described as in Proposition 5.3, and in particular for $N=4,5,6$ as in Examples 5.1, 5.2, 5.6, respectively.

\subsection{A result of Vanhove and Zerbini}

In ([51], Proposition 7.2), Vanhove and Zerbini also studied the domain of convergence of

$$
Z_{\mathbb{C}}^{(N)}(s)=\int_{\left(\mathbb{P}_{\mathbb{C}}^{1}\right)^{N-3}} \prod_{i=2}^{N-2}\left|x_{j}\right|^{2 s_{1 j}}\left|1-x_{j}\right|^{2 s_{(N-1) j}} \prod_{2 \leq i<j \leq N-2}\left|x_{i}-x_{j}\right|^{2 s_{i j}} \prod_{i=2}^{N-2} d x_{i} .
$$

Note that this integral is indeed our $Z_{\mathbb{C}}^{(N)}(s)$, since $\left(\mathbb{P}_{\mathbb{C}}^{1}\right)^{N-3}$ differs from $\mathbb{C}^{N-3}$ only by a set of measure zero and $|a|_{\mathbb{C}}=|a|^{2}$ for $a \in \mathbb{C}$. We claim that, for $N \geq 5$, the convergence domain they describe is too large. In order to explain this, we first compare their result to ours in the illustrative case $N=5$, that is,

$$
Z_{\mathbb{C}}^{(5)}(s)=\int_{\left(\mathbb{P}_{\mathbb{C}}^{1}\right)^{2}} \prod_{j=2}^{3}\left|x_{j}\right|^{2 s_{1 j}}\left|1-x_{j}\right|^{2 s_{4 j}}\left|x_{2}-x_{3}\right|^{2 s_{23}} d x_{2} d x_{3} .
$$

We established in Example 5.2 that $Z_{\mathbb{C}}^{(5)}(\boldsymbol{s})$ is convergent (and holomorphic) in the domain

$$
\left\{\begin{array}{l}
\operatorname{Re}\left(s_{12}\right)>-1, \operatorname{Re}\left(s_{13}\right)>-1, \operatorname{Re}\left(s_{42}\right)>-1, \operatorname{Re}\left(s_{43}\right)>-1, \operatorname{Re}\left(s_{23}\right)>-1 ; \\
\operatorname{Re}\left(s_{12}\right)+\operatorname{Re}\left(s_{13}\right)+\operatorname{Re}\left(s_{23}\right)>-2 ; \\
\operatorname{Re}\left(s_{42}\right)+\operatorname{Re}\left(s_{43}\right)+\operatorname{Re}\left(s_{23}\right)>-2 ; \\
\operatorname{Re}\left(s_{12}\right)+\operatorname{Re}\left(s_{42}\right)+\operatorname{Re}\left(s_{23}\right)<-1 ; \\
\operatorname{Re}\left(s_{13}\right)+\operatorname{Re}\left(s_{43}\right)+\operatorname{Re}\left(s_{23}\right)<-1 ; \\
\operatorname{Re}\left(s_{12}+s_{13}+s_{42}+s_{43}+s_{23}\right)<-2,
\end{array}\right.
$$


Now, according to [51], the integral $Z_{\mathbb{C}}^{(5)}(s)$ is convergent in the larger domain

$$
\left\{\begin{array}{l}
\operatorname{Re}\left(s_{12}\right)>-1 ; \operatorname{Re}\left(s_{13}\right)>-1 ; \operatorname{Re}\left(s_{42}\right)>-1 ; \operatorname{Re}\left(s_{43}\right)>-1 ; \operatorname{Re}\left(s_{23}\right)>-1 \\
\operatorname{Re}\left(s_{12}+s_{13}+s_{23}\right)>-2 \\
\operatorname{Re}\left(s_{43}+s_{42}+s_{23}\right)>-2 \\
\operatorname{Re}\left(s_{12}+s_{13}+s_{42}+s_{43}+s_{23}\right)<-2
\end{array}\right.
$$

i.e., (6.4) without the two inequalities on the fourth and fifth lines. In order to compare with ([51], section 7.1), we mention that they use the notation $\left(a_{1}, a_{2}, b_{1}, b_{2}, c_{12}\right)$ for $\left(s_{12}, s_{13}, s_{42}, s_{43}, s_{23}\right)$ and $k$ for $N-3$. There is a small typo in the inequalities on the second line on their (7.8), corresponding to (6.5) above. According to the outline of the proof of ([51], Proposition 7.2), the domain (6.5) is obtained by using the partition

$$
\mathbb{C}^{2}=\left\{\left(x_{2}, x_{3}\right) \in \mathbb{C}^{2} ;\left|x_{2}\right| \leq\left|x_{3}\right|\right\} \bigsqcup\left\{\left(x_{2}, x_{3}\right) \in \mathbb{C}^{2} ;\left|x_{3}\right|<\left|x_{2}\right|\right\},
$$

and performing a changes of variables of type $u=\frac{x_{2}}{x_{3}}$ and $u^{\prime}=\frac{x_{3}}{x_{2}}$ in the first and second region, respectively. In this way they derive the regions where the integral is convergent near $(0,0)$ and at infinity; the region where the integral is convergent near $(1,1)$ is then obtained by substituting $s_{1 j}$ with $s_{4 j}$.

However, the authors do not write any details about their computations, and they forget some inequalities, probably because they do not analyse the situation 'at infinity' carefully. Their last inequality is needed for convergence near $(\infty, \infty)$, and the two missing ones for convergence near $(\infty, 0)$ and $(0, \infty)$, respectively. For $(\infty, 0)$, one must perform the change of variable $x_{2} \rightarrow 1 / x_{2}$, leading in Example 5.2 to the study of convergence of the integral (5.5) around the origin, and hence to the first missing condition (5.6). Similarly, convergence near $(0, \infty)$ leads to the second missing condition (5.7).

Note that the domain (6.5) is really larger than our (6.4); for example the point $\boldsymbol{s}_{0}:=\left(s_{12}, s_{13}, s_{42}, s_{43}, s_{23}\right)=(-1 / 4,-2 / 3,-2 / 3,-2 / 3,0)$ belongs to the difference set. Then

$$
\begin{aligned}
Z_{\mathbb{C}}^{(5)}\left(\boldsymbol{s}_{0}\right) & =\int_{\mathbb{C}^{2}}\left|x_{2}\right|^{2 \cdot\left(-\frac{1}{4}\right)}\left|x_{3}\right|^{2 \cdot\left(-\frac{2}{3}\right)}\left|1-x_{2}\right|^{2 \cdot\left(-\frac{2}{3}\right)}\left|1-x_{3}\right|^{2 \cdot\left(-\frac{2}{3}\right)} d x_{2} d x_{3} \\
& =\int_{\mathbb{C}}\left|x_{2}\right|^{2 \cdot\left(-\frac{1}{4}\right)}\left|1-x_{2}\right|^{2 \cdot\left(-\frac{2}{3}\right)} d x_{2} \cdot \int_{\mathbb{C}}\left|x_{3}\right|^{2 \cdot\left(-\frac{2}{3}\right)}\left|1-x_{3}\right|^{2 \cdot\left(-\frac{2}{3}\right)} d x_{3},
\end{aligned}
$$

should be a convergent integral according to ([51], Proposition 7.2 and (7.8)). The integral in $x_{2}$ however does not converge. Indeed, a necessary condition for convergence of the integral

$$
\int_{\mathbb{C}}|x|^{-2 \alpha}|1-x|^{-2 \beta} d x
$$

is that $\alpha+\beta>1$. This is in fact part of the description of the convergence domain of $Z_{\mathbb{C}}^{(4)}(s)$ in Example 5.1 and in $([51],(7.7))$, which indeed does not contain $\left(-\frac{1}{4},-\frac{2}{3}\right)$.

More generally, for $N \geq 5$, the statement in ([51], Proposition 7.2) misses the $2^{N-3}-2$ inequalities in (5.29) with $J \subsetneq\{1, \ldots, N-3\}$. For $N=6$, these are the six inequalities of the form $\cdots<-1$ or $\cdots<-2$ in Example 5.6. 


\section{Meromorphic continuation of Koba-Nielsen string amplitudes over lo- cal fields of characteristic zero}

We set

$$
A_{\mathbb{K}}^{(N)}(\boldsymbol{k})=\left.Z_{\mathbb{K}}^{(N)}(\boldsymbol{s})\right|_{s_{i j}=\boldsymbol{k}_{i} \boldsymbol{k}_{j}},
$$

where the momenta vectors $\boldsymbol{k}_{i}$ belong to $\mathbb{C}^{l+1}$, where $l$ is an arbitrary positive integer. Typically $l$ is taken to be 25 . By Theorem $6.1, Z_{\mathbb{K}}^{(N)}(s)$ has a meromorphic continuation to the whole $\mathbb{C}^{\boldsymbol{d}}$. This section is dedicated to the study of $A_{\mathbb{K}}^{(N)}(\boldsymbol{k})$ as a meromorphic function.

Remark 4. Strictly speaking $A_{\mathbb{K}}^{(N)}(\boldsymbol{k}), \boldsymbol{k}=\left(\boldsymbol{k}_{1}, \ldots, \boldsymbol{k}_{N}\right) \in \mathbb{R}^{N(l+1)}$, is defined in the real affine set

$$
\left\{\left(\boldsymbol{k}_{1}, \ldots, \boldsymbol{k}_{N}\right) \in \mathbb{R}^{N(l+1)} ; \sum_{i=1}^{N} \boldsymbol{k}_{i}=\mathbf{0}, \quad \boldsymbol{k}_{i} \boldsymbol{k}_{i}=2 \text { for } i=1, \ldots, N\right\},
$$

where the mass of the tachyons is normalized as $m^{2}=-2$, in the case of open strings, and $m^{2}=-4$, in the case of closed strings. In Theorem 7.1 we discard the kinematic restrictions (7.2), and consider $A_{\mathbb{K}}^{(N)}(\boldsymbol{k})$ as defined in $\boldsymbol{k}=\left(\boldsymbol{k}_{1}, \ldots, \boldsymbol{k}_{N-1}\right) \in \mathbb{C}^{(N-1)(l+1)}$. At the end of this section we discuss the convergence of the string amplitudes considering the kinematic restrictions (7.2).

\subsection{Convergence of the Koba-Nielsen amplitudes}

Here we consider

$$
\boldsymbol{k}_{i}=\left(k_{0, i}, k_{1, i}, \ldots, k_{l, i}\right) \in \mathbb{C}^{l+1}, \text { for } i=1,2, \ldots, N-1 .
$$

Given two real numbers $B$ and $C$ such that $0<B<C<\frac{1}{N-2}$ and $C-B=\frac{1}{N}$, we define the open subset $\mathcal{U} \subset \mathbb{C}^{(N-1)(l+1)}$ as

$$
\mathcal{U}:=\mathcal{U}_{1} \times \cdots \times \mathcal{U}_{N-1}
$$

where each $\mathcal{U}_{i} \subset \mathbb{C}^{l+1}$ is itself an open subset defined as

$$
\mathcal{U}_{i}=\mathcal{U}_{0, i} \times \mathcal{U}_{1, i} \times \cdots \times \mathcal{U}_{l, i},
$$

where

$$
\mathcal{U}_{0, i}:=\left\{k_{0, i} \in \mathbb{C} ; \sqrt{C}<\operatorname{Re}\left(k_{0, i}\right)<\sqrt{\frac{1}{N-2}} \quad \text { and } \quad 0<\operatorname{Im}\left(k_{0, i}\right)<\sqrt{B}\right\}
$$

and

$$
\mathcal{U}_{m, i}:=\left\{k_{m, i} \in \mathbb{C} ; 0<\operatorname{Re}\left(k_{m, i}\right)<\sqrt{\frac{B}{l}} \quad \text { and } \quad \sqrt{\frac{C}{l}}<\operatorname{Im}\left(k_{m, i}\right)<\sqrt{\frac{1}{l(N-2)}}\right\},
$$

for $m=1, \ldots, l$. 
Proposition 7.1. All elements $\left(\boldsymbol{k}_{1}, \ldots, \boldsymbol{k}_{N-1}\right)$ in $\mathcal{U} \subset \mathbb{C}^{(N-1)(l+1)}$ satisfy

$$
-\frac{2}{N-2}<\operatorname{Re}\left(\boldsymbol{k}_{i} \boldsymbol{k}_{j}\right)<-\frac{2}{N} \quad \text { for all } i, j \in\{1,2, \ldots, N-1\} .
$$

Proof. By definition of the Minkowski product, we have for all $i, j$ that

$$
\boldsymbol{k}_{i} \boldsymbol{k}_{j}=-k_{0, i} k_{0, j}+\sum_{m=1}^{l} k_{m, i} k_{m, j}
$$

and hence

$$
\begin{aligned}
\operatorname{Re}\left(\boldsymbol{k}_{i} \boldsymbol{k}_{j}\right)= & -\operatorname{Re}\left(k_{0, i}\right) \operatorname{Re}\left(k_{0, j}\right)+\sum_{m=1}^{l} \operatorname{Re}\left(k_{m, i}\right) \operatorname{Re}\left(k_{m, j}\right) \\
& +\operatorname{Im}\left(k_{0, i}\right) \operatorname{Im}\left(k_{0, j}\right)-\sum_{m=1}^{l} \operatorname{Im}\left(k_{m, i}\right) \operatorname{Im}\left(k_{m, j}\right) .
\end{aligned}
$$

Now for any $\left(\boldsymbol{k}_{1}, \ldots, \boldsymbol{k}_{N-1}\right) \in \mathcal{U}$ we have

$$
0<\sum_{m=1}^{l} \operatorname{Re}\left(k_{m, i}\right) \operatorname{Re}\left(k_{m, j}\right)<B \quad \text { and } \quad C<\operatorname{Re}\left(k_{0, i}\right) \operatorname{Re}\left(k_{0, j}\right)<\frac{1}{N-2}
$$

and

$$
C<\sum_{m=1}^{l} \operatorname{Im}\left(k_{m, i}\right) \operatorname{Im}\left(k_{m, j}\right)<\frac{1}{N-2} \quad \text { and } \quad 0<\operatorname{Im}\left(k_{0, i}\right) \operatorname{Im}\left(k_{0, j}\right)<B,
$$

and thus

$$
-\frac{1}{N-2}<-\operatorname{Re}\left(k_{0, i}\right) \operatorname{Re}\left(k_{0, j}\right)+\sum_{m=1}^{l} \operatorname{Re}\left(k_{m, i}\right) \operatorname{Re}\left(k_{m, j}\right)<B-C=-\frac{1}{N}
$$

and

$$
-\frac{1}{N-2}<\operatorname{Im}\left(k_{0, i}\right) \operatorname{Im}\left(k_{0, j}\right)-\sum_{m=1}^{l} \operatorname{Im}\left(k_{m, i}\right) \operatorname{Im}\left(k_{m, j}\right)<B-C=-\frac{1}{N} .
$$

Finally (7.4) follows from (7.5) and (7.6).

\subsection{Meromorphic continuation of Koba-Nielsen string amplitudes}

Theorem 7.1. Let $\mathbb{K}$ be a local field of characteristic zero. The integral $A_{\mathbb{K}}^{(N)}(\boldsymbol{k})$ converges and is holomorphic in the open set $\mathcal{U} \subset \mathbb{C}^{(N-1)(l+1)}$. It extends to a meromorphic function in $\boldsymbol{k}$ on the whole $\mathbb{C}^{(N-1)(l+1)}$.

If $\mathbb{K}$ is an $\mathbb{R}$-field, then the possible poles of $A_{\mathbb{K}}^{(N)}(\boldsymbol{k})$ belong to

$$
\bigcup_{I \subseteq\{2, \ldots, N-2\}} \bigcup_{t \in \mathbb{N}} \bigcup_{r \in T(I)}\left\{\boldsymbol{k} \in \mathbb{C}^{(N-1)(l+1)} ; \sum_{i j \in M(I)} N_{i j, r}(I) \boldsymbol{k}_{i} \boldsymbol{k}_{j}+\gamma_{r}(I)+\frac{t}{[\mathbb{K}: \mathbb{R}]}=0\right\}
$$


where $N_{i j, r}(I), \gamma_{r}(I) \in \mathbb{Z}$, and $M(I), T(I)$ are finite sets, and $[\mathbb{K}: \mathbb{R}]=1$ if $\mathbb{K}=\mathbb{R}$, and $[\mathbb{K}: \mathbb{R}]=2$ if $\mathbb{K}=\mathbb{C}$. If $\mathbb{K}$ is a $p$-adic field, then $A_{\mathbb{K}}^{(N)}(\boldsymbol{k})$ is a rational function in the variables $q^{-\boldsymbol{k}_{i} \boldsymbol{k}_{j}}$, and its possible poles belong to

$$
\bigcup_{I \subseteq\{2, \ldots, N-2\}} \bigcup_{r \in T(I)}\left\{\boldsymbol{k} \in \mathbb{C}^{(N-1)(l+1)} ; \sum_{i j \in M(I)} N_{i j, r}(I) \operatorname{Re}\left(\boldsymbol{k}_{i} \boldsymbol{k}_{j}\right)+\gamma_{r}(I)=0\right\} .
$$

More precisely, for each $r$, either all numbers $N_{i j, r}(I)$ are equal to 0 or 1 and $\gamma_{r}(I)>0$, or all numbers $N_{i j, r}(I)$ are equal to 0 or -1 and $\gamma_{r}(I)<0$.

Proof. We consider the polynomial (and hence holomorphic) mapping

$$
M: \mathbb{C}^{(N-1)(l+1)} \rightarrow \mathbb{C}^{\boldsymbol{d}}=\mathbb{C}^{\frac{(N(N-3)}{2}}: \boldsymbol{k}=\left(\boldsymbol{k}_{1}, \ldots, \boldsymbol{k}_{N-1}\right) \mapsto \boldsymbol{s}=\left(s_{i j}\right),
$$

given by $s_{i j}=\boldsymbol{k}_{i} \boldsymbol{k}_{j}$.

By Theorem 6.1 and Proposition 7.1, we have that $M(\mathcal{U})$ is part of the region where the original integral defining the Koba-Nielsen zeta function $Z_{\mathbb{K}}^{(N)}(s)$ converges. As a consequence, the original integral defining the Koba-Nielsen amplitude $A_{\mathbb{K}}^{(N)}(\boldsymbol{k})$ converges in $\mathcal{U}$. Then, since a composition of holomorphic mappings is again holomorphic, it is clear that $A_{\mathbb{K}}^{(N)}(\boldsymbol{k})$ has a meromorphic continuation to the whole $\mathbb{C}^{(N-1)(l+1)}$, with polar locus contained in the inverse image by $M$ of the polar locus of $Z_{\mathbb{K}}^{(N)}(s)$. The description of this polar locus follows directly from Theorem 6.1.

The rationality of $A_{\mathbb{K}}^{(N)}(\boldsymbol{k})$ in the case of $p$-adic fields follows from Remark 2 .

\subsection{Tachyon scattering}

We now discuss the interaction of $N$ tachyons with momenta $\boldsymbol{k}_{1}, \ldots, \boldsymbol{k}_{N} \in \mathbb{R}^{l+1}$. We assume that the mass of each tachyon satisfies $m^{2}=-2$, for open strings, and $m^{2}=-4$, for closed strings. In this framework, $\boldsymbol{k}_{i} \boldsymbol{k}_{i}=2$ becomes the relativistic formula for the energy of the $i$-th tachyon. Then increasing $N$ means to increase the whole energy of the scattering process. This study requires finding solutions of kinematic restrictions belonging to the domain of convergence of the corresponding Koba-Nielsen integral, i.e., solutions of

$$
\begin{cases}\boldsymbol{k}_{i} \boldsymbol{k}_{j} \in\left(-\frac{2}{N-2},-\frac{2}{N}\right) \quad \text { for } \quad & \left\{\begin{array}{l}
i=1, j=2, \ldots, N-2, \text { or } \\
i=N-1, j=2, \ldots, N-2, \\
\text { or } 2 \leq i<j \leq N-2 ;
\end{array}\right. \\
\sum_{i=1}^{N} \boldsymbol{k}_{i}=\mathbf{0} ; & \text { for } \quad i=1, \ldots, N .\end{cases}
$$

(Strictly speaking, the convergence domain is larger than the region in the first line of (7.8). But this region is the realistic one to investigate uniformly in $N$.) Our efforts for finding solutions of (7.8) suggest that is unlikely to find solutions for $N$ large. In contrast, if $N \leq l+1$, then is a easy to find solutions of (7.8). We interpret this as the fact that high energy scattering processes are less probable that the ones with low energy. 
Proposition 7.2. If $N \leq l+1$, then the conditions (7.8) have solutions $\boldsymbol{k}_{1}, \ldots, \boldsymbol{k}_{N} \in \mathbb{R}^{l+1}$. Proof. We take $\boldsymbol{k}_{i}=\left(t_{i}, \overrightarrow{\boldsymbol{k}_{i}}\right)$, where $t_{i} \in \mathbb{R}$ and $\overrightarrow{\boldsymbol{k}_{i}} \in \mathbb{R}^{l}$ for $i=1, \ldots, N$, so that $\boldsymbol{k}_{i} \boldsymbol{k}_{j}=$ $-t_{i} t_{j}+\left\langle\overrightarrow{\boldsymbol{k}_{i}}, \overrightarrow{\boldsymbol{k}_{j}}\right\rangle$, where $\langle\cdot, \cdot\rangle$ denotes the standard inner product on $\mathbb{R}^{l}$. Then (7.8) becomes

$$
\begin{aligned}
\sum_{i=1}^{N} t_{i} & =0, \quad \sum_{i=1}^{N} \overrightarrow{\boldsymbol{k}_{i}}=0 ; \\
2 & =-t_{i}^{2}+\left\langle\overrightarrow{\boldsymbol{k}_{i}}, \overrightarrow{\boldsymbol{k}_{i}}\right\rangle \text { for } i=1, \ldots, N ; \\
-\frac{2}{N-2} & <-t_{i} t_{j}+\left\langle\overrightarrow{\boldsymbol{k}_{i}}, \overrightarrow{\boldsymbol{k}_{j}}\right\rangle<-\frac{2}{N},
\end{aligned}
$$

for $i, j$ as in the first line in (7.8).

We now take

$$
t_{i}=\sqrt{\frac{2}{N-1}}, \quad\left\|\overrightarrow{\boldsymbol{k}_{i}}\right\|=\sqrt{\left\langle\overrightarrow{\left.\boldsymbol{k}_{i}, \overrightarrow{\boldsymbol{k}}_{i}\right\rangle}\right.}=\sqrt{\frac{2 N}{N-1}} \text { for } i=1, \ldots, N-1
$$

such that $\left\{\overrightarrow{\boldsymbol{k}_{i}}\right\}_{1 \leq i \leq N-1}$ are two by two orthogonal. Such a choice is possible since $N-1 \leq l$, for instance

$$
\overrightarrow{\boldsymbol{k}_{i}}=\left(0, \ldots, 0, \pm \sqrt{\frac{2 N}{N-1}}, 0, \ldots, 0\right)
$$

where $\pm \sqrt{\frac{2 N}{N-1}}$ appears in the $i$-th coordinate. The conditions (7.9) dictate that $t_{N}=$ $-\sum_{i=1}^{N-1} t_{i}$ and $\overrightarrow{\boldsymbol{k}_{N}}=-\sum_{i=1}^{N-1} \overrightarrow{\boldsymbol{k}_{i}}$. We claim that (7.10) and (7.11) are satisfied. Condition (7.10) is obvious for $i=1, \ldots, N-1$. It is also satisfied for $i=N$ since

$$
-\left(\sum_{i=1}^{N-1} t_{i}\right)^{2}+\left\langle\sum_{i=1}^{N-1} \overrightarrow{\boldsymbol{k}_{i}}, \sum_{i=1}^{N-1} \overrightarrow{\boldsymbol{k}_{i}}\right\rangle=-(N-1) 2+(N-1) \frac{2 N}{N-1}=2,
$$

where we used that $\left\{\overrightarrow{\boldsymbol{k}_{i}}\right\}_{1 \leq i \leq N-1}$ are two by two orthogonal. Finally, the conditions (7.2) and (7.11) are satisfied since $\left\langle\overrightarrow{\boldsymbol{k}_{i}}, \overrightarrow{\boldsymbol{k}_{j}}\right\rangle=0$ and $-t_{i} t_{j}=-\frac{2}{N-1}$ for $1 \leq i \neq j \leq N-1$.

Next, for arbitrary $N \geq 4$, if (7.8) admits no solutions $\boldsymbol{k}_{1}, \ldots, \boldsymbol{k}_{N}$ in $\mathbb{C}^{l+1}$, the relevant question, say for $\mathbb{K}=\mathbb{R}$, becomes: does the algebraic set, determined by the kinematic restrictions (7.2), have points outside the polar locus of $A_{\mathbb{R}}^{(N)}(\boldsymbol{k})$ ?

This is a very subtle problem, for the following reason. Looking at the description of the polar locus (7.7) in Theorem 7.1, the most conceptual strategy is find a solution of (7.2) such that

$$
\sum_{i j} \boldsymbol{k}_{i} \boldsymbol{k}_{j} \notin \mathbb{Z}
$$

for all sums ranging over some non-empty subset of the $\frac{N(N-3)}{2}$ different occurring ij in $A_{\mathbb{R}}^{(N)}(\boldsymbol{k})$, i.e., all $i j$ satisfying $1 \leq i<j \leq N-1$ except $1,(N-1)$. The subtlety comes from the fact that (7.2) implies the relation

$$
\sum_{1 \leq i<j \leq N-1} \boldsymbol{k}_{i} \boldsymbol{k}_{j}=-(N-2) \in \mathbb{Z}
$$


It is a reasonable idea to search for solutions in $\mathbb{C}^{l+1}$ with $l$ as small as possible. This probably simplifies computations, and such a solution then induces automatically a solution for larger $l$, simply by putting all extra coordinates equal to zero.

When $l=1$ however, already for $N=4$ all solutions of (7.2) belong to the polar locus, as can be verified by a short computation. More precisely, for all solutions it turns out that either $\boldsymbol{k}_{1} \boldsymbol{k}_{2}=-2, \boldsymbol{k}_{2} \boldsymbol{k}_{3}=-2$, or $\boldsymbol{k}_{1} \boldsymbol{k}_{2}+\boldsymbol{k}_{2} \boldsymbol{k}_{3}=0$. Hence, they all belong to the polar locus (7.7) with $t=1$, see Example 5.1 or section 8.1 below.

When $l=2$, it is conceivable that solutions outside the polar locus exist for any $N$. We found the following family of solutions of (7.2), that lie outside the polar locus for 'many' $N$. We did not pursue this line of investigation further.

\subsubsection{Example}

We denote as before $\boldsymbol{k}_{i}=\left(t_{i}, \overrightarrow{\boldsymbol{k}_{i}}\right)$, where now $t_{i} \in \mathbb{C}$ and $\overrightarrow{\boldsymbol{k}_{i}} \in \mathbb{C}^{2}$ for $i=1, \ldots, N$. For $i=1, \ldots, N-1$ we take $t_{i}=\frac{\sqrt{-2}}{N-1}$ and $\overrightarrow{\boldsymbol{k}_{i}} \in \mathbb{R}^{2}$ with $\left\|\overrightarrow{\boldsymbol{k}_{i}}\right\|=\frac{\sqrt{2 N(N-2)}}{N-1}$, such that these $\overrightarrow{\boldsymbol{k}_{i}}$ are 'equidistributed', i.e., the angle between $\overrightarrow{\boldsymbol{k}_{i}}$ and $\overrightarrow{\boldsymbol{k}_{i+1}}$ is always $\frac{2 \pi}{N-1}$. Then $\sum_{i=1}^{N-1} \overrightarrow{\boldsymbol{k}_{i}}=\overrightarrow{0}$

Next, we take $t_{N}=-\sqrt{-2}$ and $\overrightarrow{\boldsymbol{k}_{N}}=\overrightarrow{0}$. Then (7.2) is clearly satisfied. All the $\boldsymbol{k}_{i} \boldsymbol{k}_{j}$ are of the form

$$
\frac{2}{(N-1)^{2}}+\cos \left(m \frac{2 \pi}{N-1}\right) \frac{2 N(N-2)}{(N-1)^{2}}
$$

for some positive integer $m$, bounded by $\frac{N-2}{2}$.

Using some number theoretic arguments, we verified that this solution is outside the polar locus of $A_{\mathbb{R}}^{(N)}(\boldsymbol{k})$ if $N \leq 10$, and more generally if for instance $N-1$ is a prime number.

\section{Amplitudes and gamma functions}

\subsection{Veneziano amplitude}

We recall that in the case $N=4, K=\mathbb{R}, A_{\mathbb{R}}^{(4)}(\boldsymbol{k})$ is the Veneziano amplitude

$$
A_{\mathbb{R}}^{(4)}(\boldsymbol{k})=\int_{\mathbb{R}}|x|^{\boldsymbol{k}_{1} \boldsymbol{k}_{2}}|1-x|^{\boldsymbol{k}_{2} \boldsymbol{k}_{3}} d x
$$

see e.g. [52], see also ([20], section 11), ([56], chapter 3, section XIV) and the references therein. Here the momenta $\boldsymbol{k}_{1}, \boldsymbol{k}_{2}, \boldsymbol{k}_{3}, \boldsymbol{k}_{4} \in \mathbb{R}^{l+1}$. The standard Mandelstam variables are defined as

$$
s=-\left(\boldsymbol{k}_{1}+\boldsymbol{k}_{2}\right)^{2}, \quad t=-\left(\boldsymbol{k}_{2}+\boldsymbol{k}_{3}\right)^{2}, \quad u=-\left(\boldsymbol{k}_{2}+\boldsymbol{k}_{4}\right)^{2} .
$$

They satisfy the condition $s+t+u=4 m^{2}=-8$. Notice that by using the momenta conservation condition we have $t=-\left(\boldsymbol{k}_{1}+\boldsymbol{k}_{4}\right)^{2}$ and $u=-\left(\boldsymbol{k}_{1}+\boldsymbol{k}_{3}\right)^{2}$. We set

$$
\alpha(y):=1+\frac{1}{2} y
$$


for the standard Regge trajectory. Then

$$
\begin{aligned}
A_{\mathbb{R}}^{(4)}(\boldsymbol{k})= & \int_{\mathbb{R}}|x|^{-\alpha(s)-1}|1-x|^{-\alpha(t)-1} d x= \\
& \frac{\Gamma(-\alpha(s)) \Gamma(-\alpha(t))}{\Gamma(-\alpha(s)-\alpha(t))}+\frac{\Gamma(-\alpha(t)) \Gamma(-\alpha(u))}{\Gamma(-\alpha(t)-\alpha(u))}+\frac{\Gamma(-\alpha(u)) \Gamma(-\alpha(s))}{\Gamma(-\alpha(u)-\alpha(s))},
\end{aligned}
$$

where

$$
\Gamma(z)=\int_{\mathbb{R}} x^{z-1} e^{-x} d x, \text { for } \operatorname{Re}(z)>0,
$$

is the gamma function, which is holomorphic in $\operatorname{Re}(z)>0$. This function admits a meromorphic continuation to $\mathbb{C}$ with poles in the non-positive integers. The gamma function has no zeros, hence $\frac{1}{\Gamma(z)}$ is an entire function.

By using the fact that the functions $\frac{1}{\Gamma(-\alpha(s)-\alpha(t))}, \frac{1}{\Gamma(-\alpha(t)-\alpha(u))}, \frac{1}{\Gamma(-\alpha(u)-\alpha(s))}$ are entire, $A_{\mathbb{R}}^{(4)}(\boldsymbol{k})$ is holomorphic in the solution set of

$$
\left\{\begin{aligned}
&-\alpha(s)=-1+\frac{1}{2}\left(\boldsymbol{k}_{1}+\boldsymbol{k}_{2}\right)^{2}>0 \Leftrightarrow \boldsymbol{k}_{1} \boldsymbol{k}_{2}>-1 ; \\
&-\alpha(t)=-1+\frac{1}{2}\left(\boldsymbol{k}_{2}+\boldsymbol{k}_{3}\right)^{2}>0 \Leftrightarrow \boldsymbol{k}_{2} \boldsymbol{k}_{3}>-1 ; \\
&-\alpha(u)=-1+\frac{1}{2}\left(\boldsymbol{k}_{2}+\boldsymbol{k}_{4}\right)^{2}>0 \Leftrightarrow \boldsymbol{k}_{2} \boldsymbol{k}_{4}>-1 .
\end{aligned}\right.
$$

On the other hand, by applying Theorem 5.15 and Example $5.1, A_{\mathbb{R}}^{(4)}(\boldsymbol{k})$ is holomorphic in the solution set of

$$
\boldsymbol{k}_{1} \boldsymbol{k}_{2}>-1 ; \quad \boldsymbol{k}_{2} \boldsymbol{k}_{3}>-1 ; \quad \boldsymbol{k}_{1} \boldsymbol{k}_{2}+\boldsymbol{k}_{2} \boldsymbol{k}_{3}<-1
$$

By using the momenta conservation condition $\boldsymbol{k}_{2} \boldsymbol{k}_{1}+2+\boldsymbol{k}_{2} \boldsymbol{k}_{3}+\boldsymbol{k}_{2} \boldsymbol{k}_{4}=0$, we can replace the last condition by

$$
\boldsymbol{k}_{1} \boldsymbol{k}_{2}+\boldsymbol{k}_{2} \boldsymbol{k}_{3}=-2-\boldsymbol{k}_{2} \boldsymbol{k}_{4}<-1 \Leftrightarrow \boldsymbol{k}_{2} \boldsymbol{k}_{4}>-1,
$$

which means that Example 5.1 indeed provides the exact domain of convergence of the Veneziano amplitude $A_{\mathbb{R}}^{(4)}(\boldsymbol{k})$. (We assumed implicitly that $l \geq 2$.)

\section{2 $A_{\mathbb{R}}^{(N)}(\boldsymbol{k})$ as a sum of gamma functions}

In this subsection we show that $A_{\mathbb{R}}^{(N)}(\boldsymbol{k})$ is a combination of gamma functions. Indeed,

$$
A_{\mathbb{R}}^{(N)}(\boldsymbol{k})=\sum_{I \subseteq\{2, \ldots, N-2\}} \sum_{r \in T(I)} C_{I, r}(\boldsymbol{k}) \Gamma\left(\sum_{i j \in M(I)} N_{i j, r}(I) \boldsymbol{k}_{i} \boldsymbol{k}_{j}+\gamma_{r}(I)\right)
$$

where $N_{i j, r}(I), \gamma_{r}(I) \in \mathbb{Z}$, and $M(I), T(I)$ are finite sets as in Theorem 7.1, and the $C_{I, r}(\boldsymbol{k})$ are holomorphic functions. This formula is a consequence of the fact that the meromorphic continuation of the zeta function $Z_{\mathbb{R}}^{(N)}(s)$ can be given in terms of gamma functions. This requires using the Bernstein-Sato theory, see ([38], Theorem 5.3.1 and 5.4.1). The techniques used here are contained in the proof of Theorem 5.4.1 in [38]. The formula (8.2) is obtained by providing an explicit meromorphic continuation in terms of 
gamma functions for the monomial integrals $J_{\mathbb{R}}\left(s_{1}, \ldots, s_{m}\right)$ in Lemma 6.1. We explain this construction.

Take $f(x) \in \mathbb{R}\left[x_{1}, \ldots, x_{n}\right] \backslash \mathbb{R}$. Let $b_{f}(s)$ denote the Bernstein-Sato polynomial of $f$. It is well known that all roots of this monic polynomial are negative rational numbers. Writing $b_{f}(s)=\prod_{\lambda}(s+\lambda)$, we introduce $\gamma_{f}(s):=\prod_{\lambda} \Gamma(s+\lambda)$, where $\Gamma$ is the gamma function (8.1).

Set $V:=\left\{x \in \mathbb{R}^{n} ; f(x)>0\right\}$. Given a Schwartz function $\Phi \in \mathcal{S}\left(\mathbb{R}^{n}\right)$, we consider

$$
f_{+}^{s}(\Phi):=\int_{V} f(x)^{s} \Phi(x) d x \text { for } \operatorname{Re}(s)>0 .
$$

Then it turns out that $f_{+}^{s}(\Phi)=\gamma_{f}(s) B(\Phi, s)$, where $B(\Phi, s)$ is an entire function in $s$. Furthermore for $s$ fixed, $\Phi \rightarrow B(\Phi, s)$ is a tempered distribution, cf. ([38], Theorem 5.3.1).

We use this result in the case in which $f(x)$ is a monomial. We first consider the integral

$$
I_{\theta}(s):=\int_{\mathbb{R}} \theta(y)|y|^{N s+v-1} d y=\int_{0}^{\infty}\{\theta(y)+\theta(-y)\} y^{N s+v-1} d y,
$$

where $\theta$ is a test function, $N \geq 1, v \geq 1$, and $\operatorname{Re}(s)>-\frac{v}{N}$. In this case $f(x)=x$, $b_{f}(s)=(s+1)$, and $I_{\theta}(s)$ admits a meromorphic continuation of the form

$$
I_{\theta}(s)=\Gamma(N s+v) B(\theta, s),
$$

where $B(\theta, s)$ is an entire function.

Next, we consider integrals of type

$$
\begin{aligned}
J_{\theta}\left(s_{1}, s_{2}\right) & =\int_{\mathbb{R}^{2}} \theta\left(y_{1}, y_{2}\right)\left|y_{1}\right|^{N_{1} s_{1}+v_{1}-1}\left|y_{2}\right|^{N_{2} s_{2}+v_{2}-1} d y_{1} d y_{2} \\
& =\int_{0}^{\infty} \int_{0}^{\infty} \widetilde{\theta}\left(y_{1}, y_{2}\right) y_{1}^{N_{1} s_{1}+v_{1}-1} y_{2}^{N_{2} s_{2}+v_{2}-1} d y_{1} d y_{2},
\end{aligned}
$$

with $N_{1}, N_{2} \geq 1, v_{1}, v_{2} \geq 1$, and $\operatorname{Re}\left(s_{1}\right)>-\frac{v_{1}}{N_{1}}, \operatorname{Re}\left(s_{2}\right)>-\frac{v_{2}}{N_{2}}$.

For a fixed $s_{2}$, the tempered distribution $\theta \rightarrow J_{\theta}\left(s_{1}, s_{2}\right)$ admits a Laurent expansion around $a \in \mathbb{C}$ of the form

$$
J_{\theta}\left(s_{1}, s_{2}\right)=\sum_{k_{1}=-d_{1}} C_{k_{1}}\left(\theta, s_{2}\right)\left(s_{1}-a\right)^{k_{1}}
$$

where $\theta \rightarrow C_{k_{1}}\left(\theta, s_{2}\right)$ is a tempered distribution. Now expanding $C_{k_{1}}\left(\theta, s_{2}\right)$ around $b \in \mathbb{C}$ we get

$$
J_{\theta}\left(s_{1}, s_{2}\right)=\sum_{k_{1}=-d_{1} k_{2}=-d_{2}} C_{k_{1}, k_{2}}(\theta)\left(s_{1}-a\right)^{k_{1}}\left(s_{2}-b\right)^{k_{2}},
$$

where each $\theta \rightarrow C_{k_{1}, k_{2}}(\theta)$ is a tempered distribution, cf. ([38], pg. 65-67).

Then, to determine weather or not $C_{k_{1}, k_{2}} \neq 0$, it is sufficient to show that $C_{k_{1}, k_{2}}(\theta) \neq 0$ for $\theta$ in a dense subset of $\mathcal{S}\left(\mathbb{R}^{2}\right)$. There is a dense subset in $\mathcal{S}\left(\mathbb{R}^{2}\right)$ formed by functions of type $\psi_{1}\left(y_{1}\right) \psi_{2}\left(y_{2}\right)$, where $\psi_{1}, \psi_{2} \in \mathcal{S}(\mathbb{R})$, cf. ([38], Lemma 5.4.2). Then by (8.3),

$$
J_{\psi_{1} \psi_{2}}\left(s_{1}, s_{2}\right)=\Gamma\left(N_{1} s_{1}+v_{1}\right) \Gamma\left(N_{2} s_{2}+v_{2}\right) B\left(\psi_{1} \psi_{2}, s_{1}, s_{2}\right),
$$


where $\psi_{1} \psi_{2} \rightarrow B\left(\psi_{1} \psi_{2}, s_{1}, s_{2}\right)$ is a tempered distribution, which is a holomorphic function in $\mathbb{C}^{2}$. Now given $\left(s_{1}, s_{2}\right)$, and by using the fact that the Schwartz topology is metrizable, we obtain that the functional $B\left(\psi_{1} \psi_{2}, s_{1}, s_{2}\right)$ has a unique extension to $\mathcal{S}\left(\mathbb{R}^{2}\right)$. This implies that

$$
J_{\theta}\left(s_{1}, s_{2}\right)=\Gamma\left(N_{1} s_{1}+v_{1}\right) \Gamma\left(N_{2} s_{2}+v_{2}\right) B\left(\theta, s_{1}, s_{2}\right),
$$

where $B\left(\theta, s_{1}, s_{2}\right)$ is a holomorphic function in $\mathbb{C}^{2}$.

The above argument can easily be extended to the monomial integrals given in Lemma 6.1 in the case $\mathbb{K}=\mathbb{R}, \mathbb{C}$. Finally, by using the calculations given in Example 5.2 , one obtains that $Z_{\mathbb{R}}^{(5)}(\boldsymbol{s})$ is a (very large) sum of monomial integrals of the type $J_{\mathbb{R}}\left(s_{1}, \ldots, s_{m}\right)$. Then by using the above argument, one obtains a formula of type (8.2) for $A_{\mathbb{R}}^{(5)}(s)$. We do not include it here due to the length of this formula.

\section{Acknowledgments}

The authors wish to thank Hugo García-Compeán for some discussions on string amplitudes, and Erik Panzer and Clément Dupont for calling our attention to some recent work on string amplitudes. We wish also to thank the referees for many important suggestions and questions that helped us to improve the original manuscript.

Open Access. This article is distributed under the terms of the Creative Commons Attribution License (CC-BY 4.0), which permits any use, distribution and reproduction in any medium, provided the original author(s) and source are credited.

\section{References}

[1] A. Abouelsaood, C.G. Callan Jr., C.R. Nappi and S.A. Yost, Open Strings in Background Gauge Fields, Nucl. Phys. B 280 (1987) 599 [INSPIRE].

[2] K. Aomoto, Gauss-Manin connection of integrals of difference products, J. Math. Soc. Japan 39 (1987) 191.

[3] N. Arkani-Hamed et al., Grassmannian geometry of scattering amplitudes, Cambridge University Press, Cambridge U.K. (2016).

[4] V.I. Arnold, S.M. Gussein-Zade and A.N. Varchenko, Singularités des applications différentiables. Vol II, Mir, Moscou USSR (1986).

[5] M.F. Atiyah, Resolution of Singularities and Division of Distributions, Commun. Pure Appl. Math. 23 (1970) 145.

[6] S. Balaska and T. Sahabi, Boundary states and correlation functions of tricritical Ising model from Coulomb-gas formalism, Commun. Theor. Phys. (Beijing) 51 (2009) 115.

[7] P. Belkale and P. Brosnan, Periods and Igusa local zeta functions, Int. Math. Res. Notes 49 (2003) 2655.

[8] P.M. Bleher, Analytic continuation of massless Feynman amplitudes in the Schwartz space $\mathcal{S}^{\prime}$, Rep. Math. Phys. 19 (1984) 117.

[9] I.N. Bernstein, Modules over the ring of differential operators; the study of fundamental solutions of equations with constant coefficients, Funct. Anal. Appl. 5 (1972) 89. 
[10] R. Blumenhagen, D. Lüst and S. Theisen, String Scattering Amplitudes and Low Energy Effective Field Theory, Chapter 16 in Basic in Concepts of String Theory Part of the series Theoretical and Mathematical Physics, Springer, Berlin Germany (2013), pg. 585.

[11] M. Bocardo-Gaspar, H. García-Compeán and W.A. Zúñiga Galindo, Regularization of p-adic string amplitudes, andmultivariate local zeta functions, Lett. Math. Phys. 109 (2019) 1167 [arXiv: 1611.03807] [INSPIRE].

[12] M. Bocardo-Gaspar, H. García-Compeán and W.A. Zúñiga Galindo, On p-adic string amplitudes in the limit $p$ approaches to one, JHEP 08 (2018) 043 [arXiv:1712.08725] [INSPIRE].

[13] G. Bodnár and J. Schicho, Automated resolution of singularities for hypersurfaces, J. Symb. Comput. 30 (2000) 401.

[14] C. Bogner and S. Weinzierl, Blowing up Feynman integrals, Nucl. Phys. Proc. Suppl. 183 (2008) 256 [arXiv:0806.4307] [INSPIRE].

[15] C. Bogner and S. Weinzierl, Resolution of singularities for multi-loop integrals, Comput. Phys. Commun. 178 (2008) 596 [arXiv:0709.4092] [INSPIRE].

[16] C. Bogner and S. Weinzierl, Periods and Feynman integrals, J. Math. Phys. 50 (2009) 042302 [arXiv: 0711.4863] [INSPIRE].

[17] C.G. Bollini, J.J. Giambiagi and A. González Domínguez, Analytic regularization and the divergences of quantum field theories, Nuovo Cim. 31 (1964) 550.

[18] L. Brekke, P.G.O. Freund, M. Olson and E. Witten, Nonarchimedean String Dynamics, Nucl. Phys. B 302 (1988) 365 [INSPIRE].

[19] L. Brekke and G.O.P. Freund, p-adic numbers in physics, Phys. Rept. 233 (1993) 1.

[20] J. Broedel, O. Schlotterer and F. Zerbini, From elliptic multiple zeta values to modular graph functions: open and closed strings at one loop, JHEP 01 (2019) 155 [arXiv:1803.00527] [INSPIRE].

[21] F. Brown, The Massless higher-loop two-point function, Commun. Math. Phys. 287 (2009) 925 [arXiv: 0804.1660] [INSPIRE].

[22] F. Brown and C. Dupont, Single-valued integration and superstring amplitudes in genus zero, arXiv: 1910.01107 [INSPIRE].

[23] J. Denef, Report on Igusa's Local Zeta Function, Astérisque. Vol. 201-203: Sém. Bourbaki Vol. 1990/91, Exposés 730-744 (1991) 741, pg. 359.

[24] J. Denef and F. Loeser, Motivic Igusa zeta functions, J. Alg. Geom. 7 (1998) 505 [math/9803040].

[25] J. Denef and P. Sargos, Polyèdre de Newton et distribution f $f_{+}^{s}$. I, J. Anal. Math. 53 (1989) 201.

[26] H. Elvang and Y.-t. Huang, Scattering amplitudes in gauge theory and gravity, Cambridge University Press, Cambridge U.K. (2015).

[27] P.H. Frampton and Y. Okada, The $P^{-}$adic String $N$ Point Function, Phys. Rev. Lett. 60 (1988) 484 [INSPIRE].

[28] P.G.O. Freund and M. Olson, Non-Archimedean strings, Phys. Lett. B 199 (1987) 186 [INSPIRE]. 
[29] P.G.O. Freund and E. Witten, Adelic string amplitudes, Phys. Lett. B 199 (1987) 191 [INSPIRE].

[30] H. García-Compeán, E.Y. López and W.A. Zúñiga-Galindo, p-Adic open string amplitudes with Chan-Paton factors coupled to a constant B-field, Nucl. Phys. B 951 (2020) 114904 [arXiv: 1909.09312] [INSPIRE].

[31] I.M. Gel'fand and G.E. Shilov, Generalized Functions. Vol. 1, Academic Press, New York U.S.A. (1977).

[32] D. Ghoshal and T. Kawano, Towards p-Adic string in constant B-field, Nucl. Phys. B $\mathbf{7 1 0}$ (2005) 577 [hep-th/0409311] [INSPIRE].

[33] D. Ghoshal, Exact noncommutative solitons in p-Adic strings and BSFT, JHEP 09 (2004) 041 [hep-th/0406259] [INSPIRE].

[34] P. Grange, Deformation of p-adic string amplitudes in a magnetic field, Phys. Lett. B 616 (2005) 135 [hep-th/0409305] [INSPIRE].

[35] H. Hironaka, Resolution of singularities of an algebraic variety over a field of characteristic zero, Ann. Math. 79 (1964) 109.

[36] L. Hörmander, The analysis of linear partial differential operators. I: Distribution theory and Fourier analysis, reprint of the second edition, Springer, Berlin Germany (1990).

[37] J.-I. Igusa, Tata Institute of Fundamental Research Lectures on Mathematics and Physics. Vol. 59: Lectures on Forms of higher degree, Narosa Publishing House, New Delhi India (1978).

[38] J.-I. Igusa, AMS/IP Studies in Advanced Mathematics. Vol. 14: An introduction to the theory of local zeta functions, AMS Press, Providence U.S.A. (2000).

[39] M. Kashiwara and T. Kawai, On holonomic systems for $\Pi_{l=1}^{N}\left(f_{l}+(\sqrt{ }-1) 0\right)^{\lambda_{l}}$, Publ. Res. Inst. Math. Sci. 15 (1979) 551.

[40] H. Kawai, D.C. Lewellen and S.H.H. Tye, A relation between tree amplitudes of closed and open strings, Nucl. Phys. B 269 (1986) 1 [inSPIRE].

[41] S. Kawai, Coulomb gas approach for boundary conformal field theory, Nucl. Phys. B 630 (2002) 203 [hep-th/0201146] [INSPIRE].

[42] S. Kawai, Free field realization of boundary states and boundary correlation functions of minimal models, J. Phys. A 36 (2003) 6875 [hep-th/0210032] [INSPIRE].

[43] Z. Koba and H.B. Nielsen, Reaction amplitude for $n$ mesons: A Generalization of the Veneziano-Bardakci-Ruegg-Virasora model, Nucl. Phys. B 10 (1969) 633 [INSPIRE].

[44] A. Khrennikov, S. Kozyrev and W.A. Zúñiga-Galindo, Encyclopedia of Mathematics and its Applications. Vol. 168: Ultrametric pseudodifferential equations and applications, Cambridge University Press, Cambridge U.K. (2018).

[45] E.U. Lerner and M.D. Missarov, $P^{-}$adic Feynman and String Amplitudes, Commun. Math. Phys. 121 (1989) 35 [INSPIRE].

[46] F. Loeser, Fonctions zêta locales d'Igusa à plusieurs variables, intégration dans les fibres, et discriminants, Ann. Sci. École Norm. Sup. 22 (1989) 435.

[47] M. Marcolli, Feynman motives, World Scientific, New York U.S.A. (2010). 
[48] M.L. Mendoza-Martínez, J.A. Vallejo and W.A. Zúñiga-Galindo, Acausal quantum theory for non-Archimedean scalar fields, Rev. Math. Phys. 31 (2019) 1950011 [arXiv:1805.08613] [INSPIRE].

[49] N. Seiberg and E. Witten, String theory and noncommutative geometry, JHEP 09 (1999) 032 [hep-th/9908142] [INSPIRE].

[50] E.R. Speer, Annals of Mathematics Studies. No. 62: Generalized Feynman amplitudes, Princeton University Press, Princeton U.S.A. (1969).

[51] P. Vanhove and F. Zerbini, Closed string amplitudes from single-valued correlation functions, arXiv: 1812.03018 [INSPIRE].

[52] G. Veneziano, Construction of a crossing-symmetric, Regge behaved amplitude for linearly rising trajectories, Nuovo Cim. A 57 (1968) 190 [INSPIRE].

[53] M.A. Virasoro, Alternative constructions of crossing-symmetric amplitudes with Regge behavior, Phys. Rev. 177 (1969) 2309 [InSPIRE].

[54] I.V. Volovich, p-adic string, Class. Quant. Grav. 4 (1987) L83 [InSPIRE].

[55] I.V. Volovich, Number theory as the ultimate physical theory, p-Adic. Num. Ultrametr. Anal. Appl. 2 (2010) 77.

[56] V.S. Vladimirov, I.V. Volovich and E.I. Zelenov, p-adic analysis and mathematical physics, World Scientific, Singapore (1994).

[57] E. Witten, The Feynman iє in String Theory, JHEP 04 (2015) 055 [arXiv: 1307.5124] [INSPIRE].

[58] W. Veys and W.A. Zúñiga-Galindo, Zeta functions and oscillatory integrals for meromorphic functions, Adv. Math. 311 (2017) 295 [arXiv:1510.03622].

[59] W. Veys and W.A. Zúñiga-Galindo, Zeta functions for analytic mappings, log-principalization of ideals, and Newton polyhedra, Trans. Am. Math. Soc. 360 (2008) 2205 [math/0601336].

[60] W.A. Zúñiga-Galindo, Pseudodifferential equations over non-Archimedean spaces, Lect. Notes Math. 2174 (2016) 1.

[61] W.A. Zúñiga-Galindo, B. Zambrano-Luna and E. León-Cardenal, Graphs, local zeta functions, log-Coulomb gases, and phase transitions at finite temperature, arXiv:2003.08532 [INSPIRE]. 